\title{
Stability of solitary waves in the nonlinear Dirac equation with arbitrary nonlinearity
}

\author{
Sihong Shao* \\ LMAM and School of Mathematical Sciences, Peking University, Beijing 100871, China \\ Niurka R. Quintero ${ }^{\dagger}$ \\ IMUS and Departamento de Física Aplicada I, E.S.P. Universidad de Sevilla, 41011 Sevilla, Spain \\ Franz G. Mertens $\ddagger$ \\ Physikalisches Institut, Universität Bayreuth, D-95440 Bayreuth, Germany \\ Fred Cooper ${ }^{\S}$ \\ Theoretical Division, Los Alamos National Laboratory, Los Alamos, New Mexico 87545, USA \\ and The Santa Fe Institute, 1399 Hyde Park Road, Santa Fe, New Mexico 87501, USA \\ Avinash Khare"l \\ Indian Institute of Science Education and Research, Pune 411008, India
}

Avadh Saxena ${ }^{\mathbb{I}}$

Theoretical Division and Center for Nonlinear Studies, Los Alamos National Laboratory, Los Alamos, New Mexico 87545, USA

(Received 23 May 2014; published 17 September 2014)

\begin{abstract}
We consider the nonlinear Dirac equation in $1+1$ dimension with scalar-scalar self interaction $\frac{g^{2}}{\kappa+1}(\bar{\Psi} \Psi)^{\kappa+1}$ and with mass $m$. Using the exact analytic form for rest frame solitary waves of the form $\Psi(x, t)=\psi(x) e^{-i \omega t}$ for arbitrary $\kappa$, we discuss the validity of various approaches to understanding stability that were successful for the nonlinear Schrödinger equation. In particular we study the validity of a version of Derrick's theorem and the criterion of Bogolubsky as well as the Vakhitov-Kolokolov criterion, and find that these criteria yield inconsistent results. Therefore, we study the stability by numerical simulations using a recently developed fourth-order operator splitting integration method. For different ranges of $\kappa$ we map out the stability regimes in $\omega$. We find that all stable nonlinear Dirac solitary waves have a one-hump profile, but not all one-hump waves are stable, while all waves with two humps are unstable. We also find that the time $t_{c}$, it takes for the instability to set in, is an exponentially increasing function of $\omega$ and $t_{c}$ decreases monotonically with increasing $\kappa$.

DOI: 10.1103/PhysRevE.90.032915

PACS number(s): 05.45.Yv, 11.15.Kc, 03.70.+k, 11.10.-z
\end{abstract}

\section{INTRODUCTION}

The nonlinear Dirac equation arises both in field theory and condensed matter contexts. The Bose-Einstein condensate in a honeycomb lattice in the long wavelength limit provides a specific application [1,2]. The exact spinor structure of the multicomponent condensate order parameter provides a bosonic analog to the relativisitic electrons in monolayer graphene. It has also found applications in binary optical waveguide arrays $[3,4]$ and in understanding the nonlinear dynamics in honeycomb lattices [5] including nonlinear diffraction in photonic graphene [6]. The nonlinear Dirac equation has been studied $[7,8]$ in detail in the past for the particular case that the nonlinearity parameter $\kappa=1$ (massive Gross-Neveu [9] and massive Thirring models [10]). In those studies it was found that these equations have

\footnotetext{
*sihong@math.pku.edu.cn

†niurka@us.es

${ }^{\ddagger}$ franzgmertens@gmail.com

§cooper@santafe.edu

"khare@iiserpune.ac.in

Ilavadh@1anl.gov
}

solitary wave solutions. These solutions are of the form $\Psi(x, t)=e^{-i \omega t} \psi(x)$ in the rest frame, where $\psi(x)$ is a two-component spinor.

In a recent paper [11] we generalized these solutions to arbitrary nonlinearity $\kappa$ and compared the exact solutions with the nonrelativistic reduction of these solutions. At that time there were conflicting statements about the stability of these solutions as to whether Bogolubsky's approach [12] for determining stability was valid. He suggested two approaches, one a variation of Derrick's theorem [13] which looks at stability with respect to scale transformations and suggested that for $\kappa>1$ the solitary wave should be unstable. This approach seemed to violate the continuity argument that the nonlinear Dirac (NLD) equation becomes a modified nonlinear Schrödinger (NLS) equation when $\omega$ approaches the mass parameter $m$ of the Dirac equation from below. This argument has been made more rigorous by Comech [14]. Comech (private communication) has been able to prove that for $\kappa<2$ the Vakhitov-Kolokolov [15] criterion guarantees linear stability in the nonrelativistic regime of the NLD equation for solutions of the form (in the rest frame) $\Psi(x, t)=\psi(x) e^{-i \omega t}$ where $\omega$ is less than but approximately equal to $m$. He was also able to show linear instability in the same nonrelativistic regime for $\kappa>2$. This is the first rigorous result for the Dirac 
equation that applies in the nonrelativistic regime. Below when we refer to NLS or NLD, it would be implicit that we refer to these equations with arbitrary nonlinearity $(\kappa)$.

Bogolubsky also proposed another test for determining stability based on varying the frequency $\omega$, while keeping the charge fixed. In his paper [12], Bogolubsky only used this approach for $\kappa=1$, since he believed that only at $\kappa=1$ did the stability argument based on scale transformations not apply. That argument (which we will discuss in Sec. IV), predicts that for $\kappa<1$ the solitary waves were stable under scale transformations and for $\kappa>1$ they should be unstable to scale transformations. This approach for studying stability based on varying the frequency when extended to all values of $\kappa \leqslant 2$ predicts that when $\omega \lesssim 0.7$ the solitary waves should be unstable to changes in $\omega$ for fixed charge. (Note that $\omega$ has been scaled by $m$.) We also show that the $\omega$ variational approach of Bogolubsky is equivalent to assuming that instability will occur in variational trial functions which preserve charge as we change $\omega$. Finally we will discuss the Vakhitov-Kolokolov [15] criterion as applied to the nonlinear Dirac equation. We will show that it predicts for all $\kappa<2$ that the solitary waves are stable for all values of $\omega$ and that there is a regime in $\omega$ even for $\kappa>2$ where the solitary waves are predicted to be linearly stable. However, these predictions are not confirmed by our simulations (Sec. V) which means that the Vakhitov-Kolokolov criterion is not valid for the NLD case. Before applying these methods to the NLD equation, we show that these three variational approaches to stability all give the same result when applied to the NLS equation, namely, for all values of $\omega$ when $\kappa<2$ the solutions are stable, and for $\kappa>2$ they are unstable.

Previous numerical studies of instability have been confined to the case $\kappa=1$. Bogolubsky [12] studied this problem numerically after suggesting that solitary waves of the nonlinear Dirac equation should be unstable if $\omega<\omega_{B} \approx 1 / \sqrt{2}$ for $g=1$ and $m=1$. He presented in his paper results for $\omega=0.5$ (unstable) and $\omega=0.8$ (stable) but the integration times were not given. In contrast to this, Alvarez and Soler [16] claimed based on their simulations that the solitary wave solutions for $\kappa=1$ were stable for all $\omega$ values. In our simulations, shown in the subsequent tables and figures, we find that for $\omega<\omega_{c}$ the solitary waves are metastable with a lifetime $t_{c}$ growing exponentially below $\omega_{c}$.

The integration times in Ref. [16] are much too small to observe the instabilities we have found for $\omega<\omega_{c}$. This also holds for the scattering experiments of Ref. [17] which studied the collision of two solitary waves with $\omega=0.6$ and 0.8 at $\kappa=1$. Here the former solitary wave looks stable, but the integration time is only about 100 . The simulations we have performed here have confirmed Bogolubsky's intuition that there is a critical value of $\omega$ below which the solitary waves are unstable, but they do not agree with his determination of the critical value. Our simulations are in agreement with Comech's proof [14] that in the nonrelativistic regime solitary waves should be stable for $\kappa<2$, and unstable for $\kappa>2$.

Our paper is organized as follows: in Sec. II we review the exact solution for arbitrary $\kappa$. In Sec. III we consider the nonrelativistic limit which is the nonlinear Schrödinger equation with a linear mass term. We discuss all three variational methods as applied to the NLS equation, namely,
Derrick's Theorem, stability with respect to changes in $\omega$ for fixed charge, and the Vakhitov-Kolokolov criterion.

In Sec. IV we discuss how these three approaches when applied naively lead to different conclusions for the NLD equation. A version of Derrick's theorem predicts that all solitary waves with $\kappa>1$ are unstable, which disagrees with Comech's results [14] in the nonrelativistic limit. Bogolubsky's criterion predicts that for $\omega$ less than a critical value, and $\kappa<2$, the solutions should be unstable, but in the nonrelativistic regime predicts stability. Vakhitov-Kolokolov criterion instead predicts all solutions should be stable for $\kappa<2$ and there is a domain of stability for $\omega$ smaller than a critical value where again the solution should be stable for $\kappa>2$. In Sec. V we present the results of detailed simulations of the nonlinear Dirac equation for $\kappa=1,0<\kappa<1,1<\kappa<2$, and $\kappa \geqslant 2$ and map out the stability regimes in $\omega$. For $0<\kappa \leqslant 1$ there is a stability regime for $\omega_{c} \leqslant \omega<1$, where the critical value $\omega_{c}$ increases monotonically with $\kappa$. For $1<\kappa<2$ there are two types of stability regions. For $\kappa \geqslant 2$ small stable regions exist, but only for $\kappa=2$ and values slightly larger than 2 .

We also find for $\omega<\omega_{c}$ that the time $t_{c}$ it takes for the instability to set in is an exponentially increasing function of the frequency $\omega$ and $t_{c}$ as a function of $\kappa$ decreases monotonically with increasing $\kappa$. Moreover, we find that below $\kappa=2$ there is a nonrelativistic regime of $\omega$ close to $m$ where the solitary waves are always stable. Finally, we remark that all stable NLD solitary waves have a one-hump profile, but not all one-hump waves are stable. All waves with two humps are unstable. Our conclusions are presented in Sec. VII.

\section{REVIEW OF EXACT SOLUTIONS}

The NLD equations that we are interested in are given by

$$
\left(i \gamma^{\mu} \partial_{\mu}-m\right) \Psi+g^{2}(\bar{\Psi} \Psi)^{\kappa} \Psi=0,
$$

which can be derived in a standard fashion from the Lagrangian density:

$$
\begin{aligned}
\mathcal{L} & =\left(\frac{i}{2}\right)\left[\bar{\Psi} \gamma^{\mu} \partial_{\mu} \Psi-\partial_{\mu} \bar{\Psi} \gamma^{\mu} \Psi\right]-m \bar{\Psi} \Psi+\mathcal{L}_{I} \\
\mathcal{L}_{I} & =\frac{g^{2}}{\kappa+1}(\bar{\Psi} \Psi)^{\kappa+1} .
\end{aligned}
$$

For solitary wave solutions, the field $\Psi$ goes to zero at $x \rightarrow$ $\pm \infty$. It is sufficient to go into the rest frame to discuss the solutions, since the theory is Lorentz invariant and the moving solution can be obtained by a Lorentz boost. In the rest frame we assume the wave function is of the form

$$
\Psi(x, t)=e^{-i \omega t} \psi(x) .
$$

We are interested in bound state solutions that correspond to positive energy $\omega \geqslant 0$ and which have energies in the rest frame less than the mass parameter $m$, i.e., $\omega<m$. In our previous papers $[11,18]$ we chose the representation $\gamma_{0}=\sigma_{3}$ and $i \gamma_{1}=\sigma_{1}$. Here instead, to make contact with the numerical simulation paper of Alvarez and Carreras [17] we choose the representation $\gamma^{0}=\sigma_{3}$ and $\gamma^{1}=i \sigma_{2}$.

Defining the functions $u(x), v(x), R(x), \theta(x)$ via

$$
\psi(x)=\left(\begin{array}{c}
u(x) \\
i v(x)
\end{array}\right)=R(x)\left(\begin{array}{c}
\cos \theta \\
i \sin \theta
\end{array}\right),
$$


we obtain the following equations for the spinor components $u$ and $v$ :

$$
\begin{aligned}
& \frac{d u}{d x}+(m+\omega) v-g^{2}\left(u^{2}-v^{2}\right)^{\kappa} v=0, \\
& \frac{d v}{d x}+(m-\omega) u-g^{2}\left(u^{2}-v^{2}\right)^{\kappa} u=0 .
\end{aligned}
$$

From energy-momentum conservation [11]

$$
\partial^{\mu} T_{\mu \nu}=0 ; \quad T_{\mu \nu}=\frac{i}{2}\left[\bar{\Psi} \gamma_{\mu} \partial_{\nu} \Psi-\partial_{\nu} \bar{\Psi} \gamma_{\mu} \Psi\right]-g_{\mu \nu} \mathcal{L}
$$

we obtain in the rest frame for stationary solutions

$$
T_{10}=\text { const } ; \quad T_{11}=\omega \psi^{\dagger} \psi-m \bar{\psi} \psi+\mathcal{L}_{I}=\text { const. }
$$

For solitary wave solutions vanishing at $x \rightarrow \pm \infty$ the constant in the second Eq. (7) is zero, and we obtain

$$
T_{11}=\omega \psi^{\dagger} \psi-m \bar{\psi} \psi+\mathcal{L}_{I}=0 .
$$

Multiplying the equation of motion on the left by $\bar{\Psi}$ we have that

$$
(\kappa+1) \mathcal{L}_{I}=-\omega \psi^{\dagger} \psi+m \bar{\psi} \psi-\bar{\psi} i \gamma^{1} \partial_{1} \psi,
$$

implying that $\omega \kappa \psi^{\dagger} \psi-m \kappa \bar{\psi} \psi-\bar{\psi} i \gamma^{1} \partial_{1} \psi=0$. For the Hamiltonian density we have

$$
\mathcal{H}=T_{00}=\bar{\Psi} i \gamma_{1} \partial_{1} \Psi+m \bar{\Psi} \Psi-\mathcal{L}_{I} \equiv h_{1}+h_{2}-h_{3} .
$$

Each of $h_{i}$ are positive definite. From Eqs. (8) and (9) one has the relationship:

$$
\kappa \mathcal{L}_{I}=-\bar{\psi} i \gamma^{1} \partial_{1} \psi
$$

From this we have $h_{3}=(1 / \kappa) h_{1}$, and in particular for $\kappa=1$, $\mathcal{H}=m \bar{\psi} \psi$. In terms of $R, \theta$ one has

$$
-\bar{\psi} i \gamma^{1} \partial_{1} \psi=\psi^{\dagger} \psi \frac{d \theta}{d x}
$$

This leads to the simple differential equation for $\theta$ for solitary waves:

$$
\frac{d \theta}{d x}=-\omega_{\kappa}+m_{\kappa} \cos 2 \theta ; \quad \omega_{\kappa} \equiv \kappa \omega ; \quad m_{\kappa}=\kappa m .
$$

The solution, choosing the origin of the solitary wave to be at $x=0$ (which we will do in what follows), is

$$
\theta(x)=\tan ^{-1}\left(\alpha \tanh \beta_{\kappa} x\right),
$$

where

$$
\alpha=\left(\frac{m_{\kappa}-\omega_{\kappa}}{m_{\kappa}+\omega_{\kappa}}\right)^{1 / 2}=\left(\frac{m-\omega}{m+\omega}\right)^{1 / 2}, \quad \beta_{\kappa}=\left(m_{\kappa}^{2}-\omega_{\kappa}^{2}\right)^{1 / 2} .
$$

Thus we have $\tan \theta(x)=\alpha \tanh \beta_{\kappa} x$ and

$$
\begin{aligned}
& \sin ^{2} \theta(x)=\frac{\alpha^{2} \tanh ^{2} \beta_{\kappa} x}{1+\alpha^{2} \tanh ^{2} \beta_{\kappa} x}=\frac{(m-\omega) \sinh ^{2} \beta_{\kappa} x}{m \cosh 2 \beta_{\kappa} x+\omega}, \\
& \cos ^{2} \theta(x)=\frac{1}{1+\alpha^{2} \tanh ^{2} \beta_{\kappa} x}=\frac{(m+\omega) \cosh ^{2} \beta_{\kappa} x}{m \cosh 2 \beta_{\kappa} x+\omega} .
\end{aligned}
$$

From (2) and (8) we find

$$
R^{2}=\left[\frac{(\kappa+1)(m \cos 2 \theta-\omega)}{g^{2}(\cos 2 \theta)^{\kappa+1}}\right]^{1 / \kappa} .
$$

Now we have

$$
\frac{d \theta}{d x}=\frac{\beta_{\kappa}^{2}}{\omega_{\kappa}+m_{\kappa} \cosh 2 \beta_{\kappa} x}=-\omega_{\kappa}+m_{\kappa} \cos 2 \theta .
$$

One important expression is $m \cos 2 \theta-\omega=$ $\beta_{\kappa}^{2} / \kappa^{2}\left(\omega+m \cosh 2 \beta_{\kappa} x\right)$, using which we get

$$
\begin{aligned}
R^{2}= & \left(\frac{\omega+m \cosh 2 \beta_{\kappa} x}{m+\omega \cosh 2 \beta_{\kappa} x}\right)\left[\frac{(\kappa+1) \beta_{\kappa}^{2}}{g^{2} \kappa^{2}\left(m+\omega \cosh 2 \beta_{\kappa} x\right)}\right]^{1 / \kappa} \\
= & \left(\frac{1+\alpha^{2} \tanh ^{2} \beta_{\kappa} x}{1-\alpha^{2} \tanh ^{2} \beta_{\kappa} x}\right) \\
& \times\left[\frac{\operatorname{sech}^{2} \beta_{\kappa} x(\kappa+1) \beta_{\kappa}^{2}}{g^{2}(m+\omega) \kappa^{2}\left(1-\alpha^{2} \tanh ^{2} \beta_{\kappa} x\right)}\right]^{1 / \kappa}
\end{aligned}
$$

In particular, the expressions for $R^{2}, u^{2}$, and $v^{2}$ we get for $\kappa=$ 1 agree with the expressions in Alvarez and Carreras [17] with a redefinition of the coupling to our convention. For arbitrary $\kappa$ we have (with $\beta_{\kappa}=\kappa \beta$ )

$$
\begin{aligned}
& u^{2}=\frac{(m+\omega) \cosh ^{2}(\kappa \beta x)}{m+\omega \cosh (2 \kappa \beta x)}\left\{\frac{(\kappa+1) \beta^{2}}{g^{2}[m+\omega \cosh (2 \kappa \beta x)]}\right\}^{\frac{1}{\kappa}}, \\
& v^{2}=\frac{(m-\omega) \sinh ^{2}(\kappa \beta x)}{m+\omega \cosh (2 \kappa \beta x)}\left\{\frac{(\kappa+1) \beta^{2}}{g^{2}[m+\omega \cosh (2 \kappa \beta x)]}\right\}^{\frac{1}{\kappa}} .
\end{aligned}
$$

The equation for $\omega$ in terms of $g^{2}$ is determined from the fact that the single solitary wave has charge $Q$. We have

$$
\begin{aligned}
Q & =\int_{-\infty}^{+\infty} \psi^{\dagger} \psi d x=\int_{-\infty}^{+\infty} d x R^{2}(x) \\
& =\frac{1}{\beta_{\kappa}}\left[\frac{(\kappa+1) \beta_{\kappa}^{2}}{g^{2} \kappa^{2}(m+\omega)}\right]^{1 / \kappa} I_{\kappa}\left[\alpha^{2}\right],
\end{aligned}
$$

where

$$
\begin{aligned}
I_{\kappa}\left[\alpha^{2}\right]= & \int_{-1}^{+1} d y \frac{1+\alpha^{2} y^{2}}{\left(1-y^{2}\right)^{(\kappa-1) / \kappa}\left[1-\alpha^{2} y^{2}\right]^{(\kappa+1) / \kappa}} . \\
= & B(1 / 2,1 / \kappa)_{2} F_{1}\left(1+1 / \kappa, 1 / 2,1 / 2+1 / \kappa ; \alpha^{2}\right) \\
& +\alpha^{2} B(3 / 2,1 / \kappa)_{2} F_{1}\left(1+1 / \kappa, 3 / 2,3 / 2+1 / \kappa ; \alpha^{2}\right),
\end{aligned}
$$

and ${ }_{2} F_{1}$ is a hypergeometric function and $B(p, q)$ is the $\beta$ function, also called the Eulerian integral of the first kind.

To find $\omega$ as a function of $g^{2}$ and $Q$ one solves the equation

$$
I_{\kappa}\left[\alpha^{2}\right]=Q \beta_{\kappa}\left[\frac{g^{2} \kappa^{2}(m+\omega)}{(\kappa+1) \beta_{\kappa}^{2}}\right]^{1 / \kappa} .
$$

In what follows we will scale all parameters in terms of $m$ (i.e., $\omega \rightarrow \omega / m$, etc.). For $\kappa=1, Q$ has a very simple form:

$$
Q=\int_{-\infty}^{+\infty} d x R^{2}=\frac{4 \alpha}{\left(1-\alpha^{2}\right) g^{2}}=\frac{2 \beta}{g^{2} \omega}=\frac{2 \sqrt{1-\omega^{2}}}{g^{2} \omega} .
$$


Now for $H_{1}=\int_{-\infty}^{+\infty} d x R^{2} d \theta / d x$ we have

$$
\begin{aligned}
H_{1}= & \frac{1}{\kappa}\left[\frac{\beta_{\kappa}^{2}}{m+\omega}\right]^{1+1 / \kappa}\left[\frac{\kappa+1}{\kappa^{2} g^{2}}\right]^{1 / \kappa} \int_{-\infty}^{\infty} d x \\
& \times\left[\frac{\operatorname{sech}^{2} \beta_{\kappa} x}{1-\alpha^{2} \tanh ^{2} \beta_{\kappa} x}\right]^{1+1 / \kappa} .
\end{aligned}
$$

Again changing variables, letting $y=\tanh \beta_{\kappa} x$, we obtain

$$
\begin{aligned}
H_{1} & =\frac{1}{\kappa \beta_{\kappa}}\left[\frac{\beta_{\kappa}^{2}}{m+\omega}\right]^{1+1 / \kappa}\left[\frac{\kappa+1}{\kappa^{2} g^{2}}\right]^{1 / \kappa} J_{\kappa}[\alpha], \\
J_{\kappa}[\alpha] & =\int_{-1}^{1} d y \frac{\left(1-y^{2}\right)^{1 / \kappa}}{\left(1-\alpha^{2} y^{2}\right)^{1+1 / \kappa}} \\
& =B\left(\frac{1}{2}, 1+\frac{1}{\kappa}\right){ }_{2} F_{1}\left(\frac{1}{2}, 1+\frac{1}{\kappa} ; \frac{3}{2}+\frac{1}{\kappa} ; \frac{1-\omega}{1+\omega}\right) .
\end{aligned}
$$

For $\kappa=1$,

$$
H_{1}[\kappa=1]=-\frac{2\left[\sqrt{1-\omega^{2}}-2 \tanh ^{-1}\left(\sqrt{\frac{1-\omega}{1+\omega}}\right)\right]}{g^{2}} .
$$

Now for $H_{2}=m \int_{-\infty}^{\infty} d x R^{2} \cos 2 \theta$ we have

$$
H_{2}=\left[\frac{(\kappa+1)}{\kappa^{2} g^{2}(1+\omega)}\right]^{1 / \kappa} \int_{-\infty}^{\infty} d x\left[\frac{\operatorname{sech}^{2} \beta_{\kappa} x}{1-\alpha^{2} \tanh ^{2} \beta_{\kappa} x}\right]^{1 / \kappa} .
$$

Again changing variables, letting $y=\tanh \beta_{\kappa} x$, we obtain

$$
\begin{aligned}
H_{2} & =\left[\frac{(\kappa+1)}{\kappa^{2} g^{2}(1+\omega)}\right]^{1 / \kappa}\left[\beta_{\kappa}^{2}\right]^{1 / \kappa-1 / 2} K_{\kappa}[\alpha], \\
K_{\kappa}[\alpha] & =\int_{-1}^{1} d y \frac{\left(1-y^{2}\right)^{1 / \kappa-1}}{\left(1-\alpha^{2} y^{2}\right)^{1 / \kappa}} \\
& =B\left(\frac{1}{2}, \frac{1}{\kappa}\right){ }_{2} F_{1}\left(\frac{1}{2}, \frac{1}{\kappa} ; \frac{1}{2}+\frac{1}{\kappa} ; \frac{1-\omega}{1+\omega}\right) .
\end{aligned}
$$

At $\kappa=1$,

$$
H_{2}=\frac{4 \tanh ^{-1}\left(\sqrt{\frac{1-\omega}{1+\omega}}\right)}{g^{2}} .
$$

\section{THE NONRELATIVISTIC LIMIT-NONLINEAR SCHRÖDINGER EQUATION}

In a previous paper [11] we showed that if we write the rest frame solutions as in Eqs. (5) and take the nonrelativistic limit where $(m-\omega) /(2 m) \ll 1$, then $u(x)$ obeys the equation:

$$
\omega u(x)=-\frac{1}{2 m} \frac{\partial^{2}}{\partial x^{2}} u(x)+m u(x)-g^{2}(u)^{2 \kappa+1} .
$$

Defining $\psi(x, t)=u(x) e^{-i \omega t}$ we find that $\psi(x, t)$ obeys a nonlinear Schrödinger equation with a linear term proportional to $m$ :

$$
i \frac{\partial}{\partial t} \psi+\frac{1}{2 m} \frac{\partial^{2}}{\partial x^{2}} \psi+g^{2}\left(\psi^{\star} \psi\right)^{\kappa} \psi-m \psi=0
$$

(here $\hbar=c=1$, but we keep the explicit dependence of $m$ for clarity in this section). This equation has solutions of the form $\psi(x, t)=e^{-i \omega t} \psi_{\omega}(x)$ where

$$
\psi_{\omega}(x)=A \operatorname{sech}^{1 / \kappa}\left[\beta_{k} x\right]
$$

and

$$
A^{2 \kappa}=\frac{\beta_{k}^{2}(\kappa+1)}{2 m g^{2} \kappa^{2}},
$$

and $\omega$ is given by $\omega=m-\beta_{k}^{2} / 2 m \kappa^{2}$. Thus $\beta_{k}=$ $\kappa \sqrt{2 m} \sqrt{m-\omega}$. Note that the expression for $A^{2}$ can be obtained from Eq. (19) for $R^{2}$ by letting $\alpha^{2} \rightarrow 0$ and $m+\omega \rightarrow$ $2 m$ and again in the expression for

$$
\beta_{\text {Dirac }}=\kappa \sqrt{m-\omega} \sqrt{m+\omega}
$$

by replacing $m+\omega \rightarrow 2 m$.

The analog of the "charge" (as well as the nonrelativistic limit of $Q$ in the Dirac equation) is the "mass" given by

$$
\begin{aligned}
M[\omega] & =\int d x \psi_{\omega}^{\star} \psi_{\omega}=\frac{A^{2}}{\beta_{k}} \frac{\sqrt{\pi} \Gamma\left(\frac{1}{\kappa}\right)}{\Gamma\left(\frac{1}{2}+\frac{1}{\kappa}\right)} \\
& =\left[\frac{\beta_{k}^{2}(\kappa+1)}{2 m g^{2} \kappa^{2}}\right]^{1 / \kappa} \frac{1}{\beta_{k}} \frac{\sqrt{\pi} \Gamma\left(\frac{1}{\kappa}\right)}{\Gamma\left(\frac{1}{2}+\frac{1}{\kappa}\right)} \\
& =\frac{\sqrt{\frac{\pi}{2}} \Gamma\left(\frac{1}{\kappa}\right)\left[\frac{(\kappa+1)(m-\omega)}{g^{2}}\right]^{\frac{1}{\kappa}}}{\kappa \sqrt{m} \sqrt{m-\omega} \Gamma\left(\frac{1}{2}+\frac{1}{\kappa}\right)} .
\end{aligned}
$$

\section{A. Derrick's theorem}

For the NLS equation we can use the scaling argument of Derrick [13] to determine if the solutions are unstable to scale transformation. The Hamiltonian is given by

$H=\int d x\left\{\frac{1}{2 m} \psi_{x}^{\star} \psi_{x}+m \psi^{\star} \psi-\frac{g^{2}}{\kappa+1}\left(\psi^{\star} \psi\right)^{\kappa+1}\right\}$.

From the equations of motion one can show that when we evaluate $H$ for solitary wave solutions then $H_{3}=(2 / \kappa) H_{1}$.

Thus the value of the energy of a solitary wave solution is given by

$$
H=m M\left[\psi_{\omega}\right]+\frac{\kappa-2}{2} H_{3}\left[\psi_{\omega}\right]
$$

Here

$$
\begin{aligned}
H_{3} & =\frac{g^{2}}{\kappa+1} \frac{A^{2 \kappa+2}}{\beta_{k}} \frac{\sqrt{\pi} \Gamma\left(1+\frac{1}{\kappa}\right)}{\Gamma\left(\frac{3}{2}+\frac{1}{\kappa}\right)} \\
& =\frac{\sqrt{\frac{\pi(m-\omega)}{2 m}} \Gamma\left(1+\frac{1}{\kappa}\right)\left[\frac{(\kappa+1)(m-\omega)}{g^{2}}\right]^{\frac{1}{\kappa}}}{\kappa \Gamma\left(\frac{3}{2}+\frac{1}{\kappa}\right)} .
\end{aligned}
$$

It is well known that using stability with respect to scale transformation to understand domains of stability applies to this type of Hamiltonian. This Hamiltonian can be written

$$
H=H_{1}+m H_{2}-H_{3},
$$

where $H_{i}>0(i=1,2,3)$. If we make a scale transformation on the solution which preserves the mass $M=\int \psi^{\star} \psi d x$,

$$
\psi_{\lambda} \rightarrow \lambda^{1 / 2} \psi(\lambda x)
$$


we obtain $H_{\lambda}=\lambda^{2} H_{1}+m H_{2}-\lambda^{\kappa} H_{3}$. The first derivative is

$$
\frac{\partial H}{\partial \lambda}=2 \lambda H_{1}-\kappa \lambda^{\kappa-1} H_{3} \text {. }
$$

Setting the derivative to zero at $\lambda=1$ gives the equation consistent with the equations of motion: $\kappa H_{3}=2 H_{1}$. The second derivative at $\lambda=1$ can now be written as

$$
\frac{\partial^{2} H}{\partial \lambda^{2}}=\kappa(2-\kappa) H_{3}\left[\psi_{\omega}\right]
$$

The solution is therefore unstable to scale transformations when $\kappa>2$.

\section{B. Linear stability and the Vakhitov-Kolokokov criterion}

In the case of the nonlinear Schrödinger equation, it is easy to perform a linear stability analysis for the exact solutions. Namely, one lets

$$
\psi(x, t)=\left[\psi_{\omega}(x)+r(x, t)\right] e^{-i \omega t},
$$

linearizes the equation for $r(x, t)$

$$
\partial_{t} r(x, t)=A_{\omega} r(x, t),
$$

and studies the eigenvalues of the differential operator $A_{\omega}$. If the spectrum of $A_{\omega}$ is imaginary, then the solutions are spectrally stable. Vakhitov and Kolokolov [15] showed that when the spectrum is purely imaginary, $d M[\omega] / d \omega<0$. Also they showed that when $d M[\omega] / d \omega>0$, there is a real positive eigenvalue so that there is a linear instability. For the NLS equation we have that

$$
M[\omega]=k \beta_{k}^{(2-\kappa) / \kappa}=k(m-\omega)^{(2-\kappa) /(2 \kappa)}, \quad k>0,
$$

where $k$ is positive real. Therefore

$$
\frac{d M}{d \omega}=k^{\prime}(\kappa-2) ; \quad k^{\prime}>0 .
$$

Thus for $\kappa>2$ the solitary waves are unstable.

\section{Stability to changes in the frequency at fixed charge}

In this section we will study the suggestion of Bogolubsky that we can determine stability by looking at whether the energy of the solitary wave is increased or decreased as we vary the frequency $\omega$ for fixed values of the charge. That is if we parametrize a rest frame solitary wave solution of the NLS equation, which has a charge $M[\omega]$, given by

$$
\psi_{s}(x, t)=\chi_{s}(x, \omega) e^{-i \omega t},
$$

then we choose our slightly changed wave function to be

$$
\begin{aligned}
\tilde{\psi}\left[x, t, \omega^{\prime}, \omega\right] & =\frac{\sqrt{M[\omega]}}{\sqrt{M\left[\omega^{\prime}\right]}} \chi_{s}\left(x, \omega^{\prime}\right) e^{-i \omega^{\prime} t} \\
& \equiv f\left(\omega^{\prime}, \omega\right) \chi_{s}\left(x, \omega^{\prime}\right) e^{-i \omega^{\prime} t} .
\end{aligned}
$$

Then the wave function $\tilde{\psi}\left[x, t, \omega^{\prime}, \omega\right]$ has the same charge as $\psi[x, t, \omega]$. Inserting this wave function into the Hamiltonian we get a new Hamiltonian $H_{p}$ depending on both $\omega^{\prime}, \omega$. As a function of $\omega^{\prime}$ the probe Hamiltonian $H_{p}$ is stationary at the value $\omega^{\prime}=\omega$. The probe Hamiltonian has the form

$$
\begin{aligned}
H_{p}\left[\omega^{\prime}, \omega\right]= & H_{3}\left[\omega^{\prime}\right]\left[\frac{\kappa}{2} f\left(\omega^{\prime}, \omega\right)^{2}-f\left(\omega^{\prime}, \omega\right)^{2(\kappa+1)}\right] \\
& +m M\left[\omega^{\prime}\right] f\left(\omega^{\prime}, \omega\right)^{2} .
\end{aligned}
$$

For this probe, the first derivative is identically zero for the exact solution when $\omega^{\prime}=\omega$. The second derivative with respect to $\omega^{\prime}$ evaluated at $\omega^{\prime}=\omega$ is exactly zero at $\kappa=2$, it is then positive for all $\omega$ for $\kappa<2$ and strictly negative for all $\omega$ for $\kappa>2$. Thus this test agrees with all the other variational methods in giving instability for all $\omega$ when $\kappa>2$. It has nothing to say at the critical value $\kappa=2$.

The second derivative evaluated at $\omega^{\prime}=\omega$ is explicitly given by

$$
\left.\frac{\partial^{2} H_{p}\left[\omega^{\prime}, \omega\right]}{\partial \omega^{\prime 2}}\right|_{\omega^{\prime}=\omega}=\frac{\sqrt{\pi}(2-\kappa)(\kappa+1)^{\frac{1}{\kappa}}(m-\omega)^{\frac{1}{\kappa}-1} \Gamma\left(1+\frac{1}{\kappa}\right)}{4 \sqrt{2-2 \omega} \Gamma\left(\frac{3}{2}+\frac{1}{\kappa}\right)} .
$$

\section{VARIATIONAL APPROACHES TO THE STABILITY OF EXACT SOLUTIONS OF THE NONLINEAR DIRAC EQUATION}

In this section we will investigate whether the variational methods that were successful in determining the domain of stability in the nonrelativistic regime could be extended to the full relativistic regime $(\omega<m)$ of the NLD equation. We will show that these three approaches suggest totally different answers as to the domain of stability as a function of $\omega$.

\section{A. Stability to scale transformations at fixed charge}

The first approach to stability (Derrick [13]) is to consider how the energy of the solitary wave responds to the scale transformation $x \rightarrow \lambda x$ [12]. The exact solution is a stationary value of $H_{\lambda}$ when $\lambda=1$ with the constraint that the charge is kept fixed. The assumption of this approach is that if the second derivative is negative at $\lambda=1$ then the solutions are unstable to scale transformations and thus unstable.

Bogolubsky applied this argument to the Dirac equation and obtained a result, which we will present, that suggests that for the NLD equation for $\kappa>1$ the solitary waves are unstable. This disagrees with our intuition, presented in Ref. [11] that in the nonrelativistic regime the NLD solitary waves should obey the same pattern of instability as the NLS equation. This intuition has been given more credence in the recent linear stability analysis of the NLD equation by Comech [14], which relies on studying the NLD equation in the nonrelativistic regime. In that study, it was found that in the nonrelativistic regime, the stability of the NLD equation solitary waves should go over to the NLS equation result that for $\kappa<2$ the solitary waves are stable. Our numerical evidence supports this analysis.

The solution is of the form

$$
\psi(x)=\left(\begin{array}{c}
u \\
i v
\end{array}\right)=R(x)\left(\begin{array}{c}
\cos \theta \\
i \sin \theta
\end{array}\right) .
$$

If we want to keep the charge fixed we consider the following stretched solution:

$$
\psi_{\lambda}(x)=\left(\begin{array}{c}
u \\
i v
\end{array}\right)=\lambda^{\frac{1}{2}} R(\lambda x)\left(\begin{array}{c}
\cos \theta(\lambda x) \\
i \sin \theta(\lambda x)
\end{array}\right) .
$$


The value of the Hamiltonian

$$
\begin{aligned}
H & =\int d x\left[\bar{\psi} i \gamma^{1} \partial_{1} \psi+m \bar{\psi} \psi-\frac{g^{2}}{\kappa+1}(\bar{\psi} \psi)^{\kappa+1}\right] \\
& \equiv H_{1}+H_{2}-H_{3},
\end{aligned}
$$

for the stretched solution is $H_{\lambda}=\lambda H_{1}+H_{2}-\lambda^{\kappa} H_{3}$, where again $H_{i}$ are all positive definite. The first derivative is

$$
\frac{\partial H_{\lambda}}{\partial \lambda}=H_{1}-\kappa \lambda^{\kappa-1} H_{3} .
$$

At the stationary point, setting $\lambda=1$, we obtain $H_{3}=$ $(1 / \kappa) H_{1}$, which is consistent with the equation of motion result we obtained earlier $\left(h_{3}=h_{1} / \kappa\right)$. We see that for $\kappa=1$ the energy is given by just $\mathrm{H}_{2}$. The second derivative yields

$$
\frac{\partial^{2} H_{\lambda}}{\partial \lambda^{2}}=-\kappa(\kappa-1) \lambda^{\kappa-2} H_{3}
$$

From this we see that if $\kappa>1$, this analysis would suggest that solitary waves are unstable to small changes in the width. For $\kappa<1$ the solitary waves are stable to this type of perturbation. The case $\kappa=1$ would require a separate treatment since this analysis yields no information.

The weakness in this argument is that one needs to prove that the stable solutions of the NLD equation are not merely stationary solutions of the variational principle but are actually minima of $H_{\lambda}$. The fact that this idea disagrees both with the continuity argument of Comech [14] and our simulations makes us seriously doubt this assumption. We find that even at $\kappa=2$ there is a range of $\omega$ near $m$ where the solitary waves are stable.

\section{B. Stability to changes in the frequency at fixed charge}

Bogolubsky [12] also suggested that the stability could be ascertained by looking at variations of the wave function, keeping the charge fixed and seeing if the solution was a minimum or maximum of the Hamiltonian as a function of the parameter $\omega$. If the deformed solution decreases the energy, then he assumed that this is a sufficient condition for the solitary wave to be unstable. Bogolubsky applied this criterion only for the case $\kappa=1$ since he presumably thought that Derrick's theorem was applicable at all other values of $\kappa$.

We know the wave function $\psi$ for the solitary wave at the value of $\omega$ corresponding to a fixed charge $Q$. If we change the parametric dependence of $\psi$ on $\omega$ this also changes the charge. This can be corrected by assuming that the new wave function has a new normalization that corrects for this. That is if we parametrize a rest frame solitary wave, which has a charge $Q[\omega]$ given by Eq. (21), as

$$
\psi_{s}(x, t)=\chi_{s}(x, \omega) e^{-i \omega t},
$$

then we choose our slightly changed wave function to be

$$
\begin{aligned}
\tilde{\psi}\left[x, t, \omega^{\prime}, \omega\right] & =\frac{\sqrt{Q[\omega]}}{\sqrt{Q\left[\omega^{\prime}\right]}} \chi_{s}\left(x, \omega^{\prime}\right) e^{-i \omega^{\prime} t} \\
& \equiv f\left(\omega^{\prime}, \omega\right) \chi_{s}\left(x, \omega^{\prime}\right) e^{-i \omega^{\prime} t} .
\end{aligned}
$$

The wave function $\tilde{\psi}\left[x, t, \omega^{\prime}, \omega\right]$ has the same charge as $\psi[x, t, \omega]$. Inserting this wave function into the Hamiltonian we get a new Hamiltonian $H_{p}$ depending on both $\omega^{\prime}, \omega$. As a function of $\omega^{\prime}$ the probe Hamiltonian $H_{p}$ is stationary at the value $\omega^{\prime}=\omega$. The criterion Bogolubsky proposes is that the solitary wave is unstable to this type of perturbation if the probe Hamiltonian has a maximum at $\omega^{\prime}=\omega$. What we will find using this approach is that the second derivative of the probe Hamiltonian is negative below a critical value of $\omega$, where $\omega_{B} \approx 0.7$, suggesting an instability for all $\omega$ less than this value. For $\kappa \leqslant 2$ using this criterion we find a regime near $\omega=m$ where $\omega<m$ and the second derivative is positive, suggesting stability in the nonrelatistic regime in agreement with Comech [14]. We will use the notation $\omega_{B}$ for the critical value of $\omega$ below which the Bogolubsky criterion leads to instability.

The probe Hamiltonian has the form:

$$
\begin{aligned}
H_{p}\left[\omega^{\prime}, \omega\right]= & H_{1}\left[\omega^{\prime}\right]\left[f\left(\omega^{\prime}, \omega\right)^{2}-\frac{1}{\kappa} f\left(\omega^{\prime}, \omega\right)^{2(\kappa+1)}\right] \\
& +H_{2}\left[\omega^{\prime}\right] f\left(\omega^{\prime}, \omega\right)^{2} .
\end{aligned}
$$

In what follows we will suppress the dependence of $H_{p}$ on $g$ since that dependence is multiplicative, namely, $H_{p} \propto \frac{1}{g^{2 \kappa}}$. For all values of $\kappa$ we find that the first derivative of $H_{p}$ with respect to $\omega^{\prime}$ evaluated at $\omega^{\prime}=\omega$ is indeed zero. The behavior of the second derivative evaluated at $\omega^{\prime}=\omega$ as a function of $\omega$, is different as we change $\kappa$. For $\kappa<2$ the second derivative becomes negative for $\omega<\omega_{B} \approx 0.7$ and becomes positive above that value. This is seen in Fig. 1 for $\kappa=1$.

For $\kappa>2$ there is a second regime near the nonrelativistic limit where the second derivative again becomes negative. For example when $\kappa=5 / 2$ the second derivative becomes negative both for $\omega<0.699276$ and in the nonrelativistic regime $\omega>0.902641$. This is shown in Fig. 2. This is in accord with the fact that for $\kappa>2$ the NLS solutions are unstable to blowup. However, note that there is a range of $\omega$ where the second derivative is positive where stability is not ruled out by this criterion.

For $\kappa=1$ we have that

$$
f\left(\omega^{\prime}, \omega\right)^{2}=\frac{\beta[\omega] \omega^{\prime}}{\beta\left[\omega^{\prime}\right] \omega},
$$

where $\beta[\omega]=\sqrt{1-\omega^{2}}$. The first derivative of $H_{p}$ with respect to $\omega^{\prime}$ evaluated at $\omega^{\prime}=\omega$ is zero. The second derivative evaluated at $\omega^{\prime}=\omega$ leads to the following expression:

$\left.\frac{\partial^{2} H_{p}\left[\omega^{\prime}, \omega\right]}{\partial \omega^{\prime 2}}\right|_{\omega^{\prime}=\omega}=-\frac{2\left[\sqrt{1-\omega^{2}}\left(\omega^{2}-3\right)+4 \tanh ^{-1}\left(\sqrt{\frac{1-\omega}{\omega+1}}\right)\right]}{\omega^{2}\left(\omega^{2}-1\right)^{2}}$.

This function is zero at $\omega_{B}=0.697586$, and the second derivative is negative below this value of $\omega$ (see Fig. 1). The values of $\omega_{B}$ vary very slightly with $\kappa$. We find

$$
\begin{array}{lll}
\omega_{B}=0.703714 & \text { for } \quad \kappa=1 / 10 ; \\
\omega_{B}=0.699767 & \text { for } \quad \kappa=1 / 3, \\
\omega_{B}=0.698531 & \text { for } \quad \kappa=1 / 2 ; \\
\omega_{B}=0.697586 & \text { for } \quad \kappa=1, \\
\omega_{B}=0.697963 & \text { for } \quad \kappa=3 / 2 ; \\
\omega_{B}=0.698612 & \text { for } \quad \kappa=2 .
\end{array}
$$




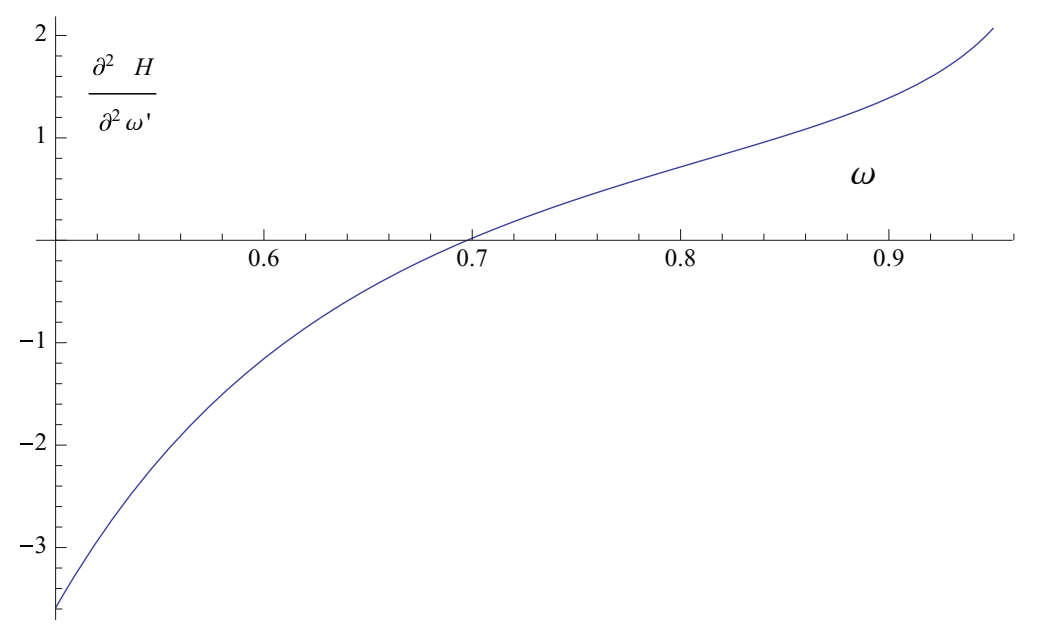

FIG. 1. (Color online) Second derivative of probe Hamiltonian at $\omega^{\prime}=\omega$ as a function of $\omega$ for $\kappa=1$.

One can view the probe Hamiltonian in a slightly different fashion. Suppose we were choosing trial wave functions which have a fixed charge $Q=1$ in a time-dependent variational approach to the problem. Then we would choose as our trial wave functions to be

$$
\psi_{v}=\frac{\psi[\omega]}{\sqrt{Q\left[\omega, g^{2}\right]}} .
$$

Here $Q\left[\omega, g^{2}\right]=\int d x \psi^{\dagger} \psi$. We would now find that the new Hamiltonian is given by

$$
H_{v}\left[\omega, g^{2}\right]=H_{1}[\omega]\left\{\frac{1}{Q\left[\omega, g^{2}\right]}-\frac{1}{\kappa}\left(\frac{1}{Q\left[\omega, g^{2}\right]}\right)^{\kappa+1}\right\}+\frac{H_{2}[\omega]}{Q\left[\omega, g^{2}\right]} .
$$

Thinking now of $\omega$ as a variational parameter to be determined by the minimization of this Hamiltonian we would next determine $\omega$ as a function of $g^{2}$ by finding the stationary value of this Hamiltonian.

As an example let us choose $\kappa=1$, where $\omega$ for fixed charge $Q$ is a function of $g^{2}$. Then

$$
H_{v}\left[\omega, g^{2}\right]=-\frac{g^{2} \omega\left[\left(\omega^{2}-1\right)\left(g^{2} \omega-2 \sqrt{1-\omega^{2}}\right)+2\left(g^{2} \omega \sqrt{1-\omega^{2}}+4 \omega^{2}-4\right) \tanh ^{-1}\left(\sqrt{\frac{1-\omega}{1+\omega}}\right)\right]}{2\left(1-\omega^{2}\right)^{3 / 2}} .
$$

The first derivative is zero when $g^{2}[\omega]$ is given by Eq. (24), i.e.,

$$
g^{2}[\omega]=\frac{2 \sqrt{1-\omega^{2}}}{\omega}
$$

Also the second derivative of this Hamiltonian, evaluated at $g^{2}[\omega]$ changes sign exactly at $\omega_{B}=0.697586$. This approach can be shown to be equivalent to the Bogolubsky approach and yields the same values of $\omega_{B}$.

\section{Vakhitov-Kolokolov criterion}

In this section we will study the consequences of assuming that the Vakhitov-Kolokolov criterion, which was derived for the NLS equation, holds for the whole range of $\omega$ in the NLD case. That is we will explore the consequences of assuming one has stability when

$$
\frac{d Q[\omega]}{d \omega}<0
$$

and instability otherwise. For the NLD equation one has that

$$
\begin{aligned}
Q[\omega]= & \frac{\sqrt{\pi}((\kappa+1)(1-\omega))^{\frac{1}{\kappa}} \Gamma\left(1+\frac{1}{\kappa}\right)}{\kappa \omega(\omega+1) \sqrt{1-\omega^{2}}} \\
& \times(\kappa+1)(\omega+1){ }_{2} F_{1}\left(-\frac{1}{2}, 1+\frac{1}{\kappa} ; \frac{3}{2}+\frac{1}{\kappa} ; \frac{1-\omega}{1+\omega}\right) \\
& +\omega(-\kappa+\omega-1){ }_{2} F_{1}\left(\frac{1}{2}, 1+\frac{1}{\kappa} ; \frac{3}{2}+\frac{1}{\kappa} ; \frac{1-\omega}{1+\omega}\right),
\end{aligned}
$$

where ${ }_{2} F_{1}$ is a hypergeometric function. Taking the derivative, we find that for $\kappa<2$ it is always negative, suggesting that the solitary waves are stable in the entire range of $\omega$ values, i.e., $0<\omega<1$. For $\kappa>2$ one finds that there is a region of $\omega$ below the curve $\omega^{\star}(\kappa)$ where the solitary waves are suggested to be stable (Fig. 3). However, both suggestions will not be confirmed by our simulations (Sec. V). Thus the VakhitovKolokolov criterion is not valid for the NLD case. 


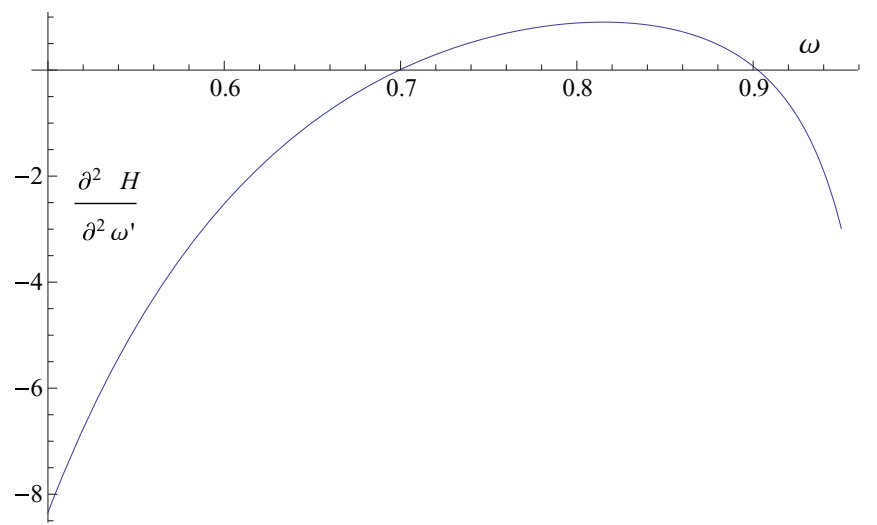

FIG. 2. (Color online) Second derivative of probe Hamiltonian at $\omega^{\prime}=\omega$ as a function of $\omega$ for $\kappa=5 / 2$.

\section{NUMERICAL METHODS}

We have shown that different theoretical methods lead to different results on the stability of NLD solitary waves. In order to understand and resolve these inconsistent results, we try to study numerically the stability of NLD solitary waves. We first tried a fourth-order Runge-Kutta method which had worked very well for forced NLS equations with arbitrary nonlinearity exponent $\kappa$ [19]. However, for the NLD equation we obtained inconsistent results, in particular for small values of $\kappa$. Various other numerical methods have been proposed in solving the NLD equation, and readers are referred to a recent review [20]. It is also reported there that the operator splitting (OS) method performs better than other numerical methods in terms of accuracy and efficiency. The main advantage of the OS method is that different numerical techniques can be exploited into integrating the subproblems in view of the features of the subproblems. In this work, we will employ the OS method to investigate the stability of NLD solitary waves. The NLD system is decomposed into two subproblems: one is linear and the other one is nonlinear, and both of them can be integrated analytically with the nonreflection boundary condition (NRBC). For the sake of completeness, we will briefly describe below the OS scheme used in this paper; the

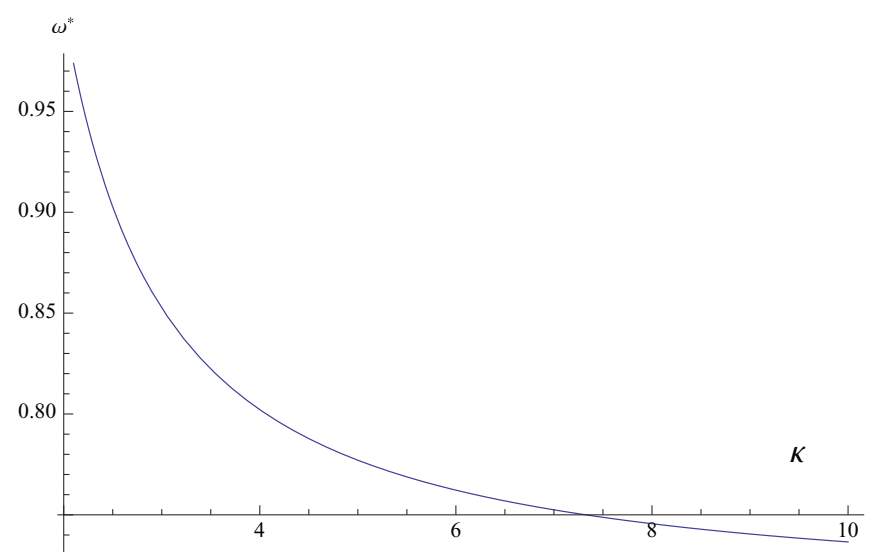

FIG. 3. (Color online) $\omega^{\star}$ as a function of $\kappa$. For $\omega \leqslant \omega^{\star}$, $d Q / d \omega<0$ and there is no instability predicted for this deformation in this regime of $\omega$. related detailed theoretical analysis and numerical comparison with other schemes can be found in Ref. [20].

For convenience, we rewrite the NLD system into

$$
\boldsymbol{\Psi}_{t}=(\mathcal{L}+\mathcal{N}) \boldsymbol{\Psi}
$$

where the linear operator $\mathcal{L}$ and the nonlinear operator $\mathcal{N}$ are defined by

$$
\mathcal{L} \boldsymbol{\Psi}:=-\boldsymbol{\gamma}^{0} \boldsymbol{\gamma}^{1} \boldsymbol{\Psi}_{x}, \quad \mathcal{N} \boldsymbol{\Psi}:=i(f-m) \boldsymbol{\gamma}^{0} \boldsymbol{\Psi}
$$

with $f:=g^{2} w^{\kappa}$ and $w:=\overline{\boldsymbol{\Psi}} \boldsymbol{\Psi}$. In consequence, the problem (69) may be decomposed into two subproblems as follows:

$$
\begin{aligned}
& \boldsymbol{\Psi}_{t}=\mathcal{L} \boldsymbol{\Psi}, \\
& \boldsymbol{\Psi}_{t}=\mathcal{N} \boldsymbol{\Psi} .
\end{aligned}
$$

Due to the local conservation law [see Eq. (78) below] the solution of the nonlinear subproblem (71) may be expressed as an exponential of the operator $\mathcal{N}$ acting on "initial data." Thus we may introduce the exponential operator splitting scheme for the NLD equation (69), imitating that for the linear partial differential equations. Based on the exact or approximate solvers of those two subproblems, a more general $K$-stage $N$ th-order exponential operator splitting method [21] for the system (69) evolving from the $n$th step to the $n+1$-th step can be cast into a product of finitely many exponentials as follows:

$$
\boldsymbol{\Psi}_{j}^{n+1}=\prod_{i=1}^{K}\left[\exp \left(\tau_{i} \mathcal{A}_{i}^{(1)}\right) \exp \left(\tau_{i} \mathcal{A}_{i}^{(2)}\right)\right] \boldsymbol{\Psi}_{j}^{n},
$$

where $\tau_{i}=a_{i} \tau$, with $\tau>0$ being the time step size, denotes the time step size used within the $i$ th stage and satisfies $\sum_{i=1}^{K} a_{i}=1$, and $\left\{\mathcal{A}_{i}^{(1)}, \mathcal{A}_{i}^{(2)}\right\}$ is any permutation of $\{\mathcal{L}, \mathcal{N}\}$. The classical second-order Strang method [22] can be represented by $\widehat{1}_{2} \frac{1}{2}^{T}$ (i.e., $a_{i}=\frac{1}{2}$ for $i=1,2$ ) if denoting $\widehat{a}_{i}:=$ $\mathrm{e}^{\tau_{i} \mathcal{A}_{i}^{(1)}} \mathrm{e}^{\tau_{i} \mathcal{A}_{i}^{(2)}}$ and $\widehat{a}_{i}^{T}:=\mathrm{e}^{\tau_{i} \mathcal{A}_{i}^{(2)}} \mathrm{e}^{\tau_{i} \mathcal{A}_{i}^{(1)}}$ [21]. The remaining task is to determine the operators $\mathrm{e}^{\tau_{i} \mathcal{L}}$ and $\mathrm{e}^{\tau_{i} \mathcal{N}}$, i.e., the solvers of the subproblems.

The computational domain is set to be $\left[0, t_{\text {fin }}\right] \times\left[X_{L}, X_{R}\right]$. Let $t_{n}=n \tau \quad\left(n=0,1, \ldots, t_{\mathrm{fin}} / \tau\right)$ and $x_{j}=X_{L}+(j-1) h$ $(j=1,2, \ldots, J)$ with $x_{J}=X_{R}$. The ghost points are denoted by $x_{0}$ and $x_{J+1}$. Here $\tau$ and $h$ are the time spacing and the spatial spacing, respectively.

\section{A. Linear subproblem}

We now solve the linear subproblem (70). We denote its "initial data" by $\boldsymbol{\Psi}_{j}^{(0)}=\left(\left(\psi_{1}\right)_{j}^{(0)},\left(\psi_{2}\right)_{j}^{(0)}\right)^{T}$ at the $i$ th stage in (72) and its solution after $\tau_{i}$ by $\boldsymbol{\Psi}_{j}^{(1)}=\left(\left(\psi_{1}\right)_{j}^{(1)},\left(\psi_{2}\right)_{j}^{(1)}\right)^{T}$. Denoting $\phi_{1}=\psi_{1}+\psi_{2}$ and $\phi_{2}=\psi_{1}-\psi_{2}$, the linear subproblem (70) can be rewritten as

$$
\begin{gathered}
\partial_{t} \phi_{1}+\partial_{x} \phi_{1}=0, \\
\partial_{t} \phi_{2}-\partial_{x} \phi_{2}=0,
\end{gathered}
$$

which means that the initial data of $\phi_{1}$ (resp. $\phi_{2}$ ) simply propagate unchanged to the right (resp. left) with velocity 1 . Therefore (73) can be exactly integrated by the characteristics 
TABLE I. Accuracy check for the OS method with NRBC and a rational fourth-order splitting. We take a normalized solitary wave with $\kappa=1$ and $\omega=0.50$ as an example, and measure the related quantities within the domain $[-100,100]$ at $t=100$. Here $\tau$ is the time step size, $\operatorname{err}_{2}$ and $\operatorname{err}_{\infty}$ are, respectively, the $l^{2}$ and $l^{\infty}$ errors, $q$ denotes the centroid position of charge density, $\mathcal{V}_{Q}, \mathcal{V}_{E}, \mathcal{V}_{P}$ measure, respectively, the variation of charge, energy, and linear momentum at the final time relative to the initial quantities.

\begin{tabular}{lcccccccc}
\hline \hline$\tau$ & $\operatorname{err}_{2}$ & Order & $\operatorname{err}_{\infty}$ & Order & $q$ & $\mathcal{V}_{Q}$ & $\mathcal{V}_{E}$ \\
\hline 0.1 & $2.99 \mathrm{E}-09$ & & $2.12 \mathrm{E}-09$ & & $2.75 \mathrm{E}-14$ & $2.22 \mathrm{E}-16$ & $2.22 \mathrm{E}-16$ \\
0.05 & $1.86 \mathrm{E}-10$ & 4.01 & $1.32 \mathrm{E}-10$ & 4.01 & $3.20 \mathrm{E}-15$ & $1.78 \mathrm{E}-14$ & $8.33 \mathrm{E}-15$ \\
0.025 & $1.16 \mathrm{E}-11$ & 4.00 & $8.24 \mathrm{E}-12$ & 4.00 & $1.33 \mathrm{E}-14$ & $7.66 \mathrm{E}-15$ & $3.44 \mathrm{E}-15$ & $2.30 \mathrm{E}-16$ \\
0.0125 & $7.26 \mathrm{E}-13$ & 4.00 & $5.87 \mathrm{E}-13$ & 3.81 & $1.23 \mathrm{E}-14$ & $1.14 \mathrm{E}-13$ & $5.55 \mathrm{E}-14$ & $2.98 \mathrm{E}-16$ \\
\hline \hline
\end{tabular}

method with $\tau_{i}=h$ as follows:

$$
\begin{aligned}
& \left(\phi_{1}\right)_{j}^{(1)}=\left(\phi_{1}\right)_{j-1}^{(0)}, \\
& \left(\phi_{2}\right)_{j}^{(1)}=\left(\phi_{2}\right)_{j+1}^{(0)},
\end{aligned}
$$

with $j=1, \cdots, J$, and the values at the ghost points are naturally given by NRBC as

$$
\begin{gathered}
\left(\phi_{1}\right)_{0}:=\phi_{1}\left(x_{0}, t\right)=0, \\
\left(\phi_{2}\right)_{J+1}:=\phi_{2}\left(x_{J+1}, t\right)=0,
\end{gathered}
$$

where we have merely used the fact that outside a relatively big domain $\left[X_{L}, X_{R}\right]$, the NLD spinor $\boldsymbol{\Psi}$ is negligibly small for it decays exponentially as $|x| \rightarrow+\infty$. Consequently, we obtain the solution $\Psi_{j}^{(1)}=\left(\left(\psi_{1}\right)_{j}^{(1)},\left(\psi_{2}\right)_{j}^{(1)}\right)^{T}$ of the following form:

$$
\begin{aligned}
\left(\psi_{1}\right)_{j}^{(1)} & =\frac{\left(\phi_{1}\right)_{j}^{(1)}+\left(\phi_{2}\right)_{j}^{(1)}}{2}, \\
{[} & \\
\left(\psi_{2}\right)_{j}^{(1)} & =\frac{\left(\phi_{1}\right)_{j}^{(1)}-\left(\phi_{2}\right)_{j}^{(1)}}{2} .
\end{aligned}
$$

The characteristic method is very appropriate for the linear subproblem (70) only under the condition of $\frac{\tau_{i}}{h}$ to be an integer for all $i=1, \ldots, K$, i.e., all $a_{i}$ must be rational. That is, the spatial spacing $h$ should be smaller than the time spacing $\tau$, which results in huge computational cost. For example, a fourth-order splitting with rational $a_{i}$ demands 18 stages given in Ref. [21]:

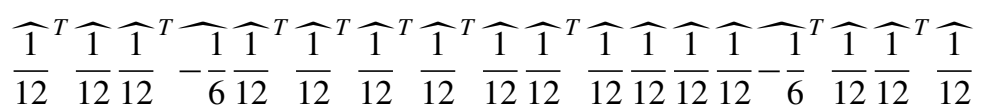

and requires that $h=\frac{1}{12} \tau$, which implies that the number of grid points is $J=96000$ if choosing $\tau=0.025$ and $-X_{L}=$ $X_{R}=100$. To accelerate the simulations, we will adopt the multithread technology provided by OpenMP. Note in passing that numerical results for the OS method are reported only for periodic boundary conditions with an irrational fourth-order splitting [20].

\section{B. Nonlinear subproblem}

The nonlinear subproblem (71) is left to be solved now. Its "initial data" are still denoted by $\boldsymbol{\Psi}_{j}^{(0)}=\left(\left(\psi_{1}\right)_{j}^{(0)},\left(\psi_{2}\right)_{j}^{(0)}\right)^{T}$ at the $i$ th stage in (72), and define

$$
t_{n}^{(i)}=t_{n}+\sum_{p=1}^{i-1} \tau_{p}, \quad i=1,2, \ldots, K
$$

For the nonlinear subproblem (71), it is not difficult to verify that

$$
\partial_{t} w=0, \quad \partial_{t} f=0
$$

Using this local conservation law gives analytically the solution at $t=t_{n}^{(i+1)}$ of (71) with the "initial data" $\boldsymbol{\Psi}_{j}^{(0)}$ as follows:

$$
\begin{aligned}
\boldsymbol{\Psi}_{j}^{(1)}= & \exp \left(i \int_{t_{n}^{(i)}}^{t_{n}^{(i+1)}}(f-m)_{j} \boldsymbol{\gamma}^{0} d t\right) \boldsymbol{\Psi}_{j}^{(0)} \\
= & \exp \left[i(f-m)_{j}^{(0)} \boldsymbol{\gamma}^{0} \tau_{i}\right] \boldsymbol{\Psi}_{j}^{(0)} \\
= & \operatorname{diag}\left\{\exp \left[i(f-m)_{j}^{(0)} \tau_{i}\right]\right. \\
& \left.\times \exp \left[-i(f-m)_{j}^{(0)} \tau_{i}\right]\right\} \boldsymbol{\Psi}_{j}^{(0)} .
\end{aligned}
$$

With NRBC, subproblems (70) and (71) can be both solved analytically, and the numerical error only comes from the operator splitting in time. That is, the OS method with the rational splitting (77) (recall that the spatial spacing $h=\frac{\tau}{12}$ ), denoted by $\mathrm{OS}(4)$ hereafter, is of the order $O\left(\tau^{4}\right)$, which is confirmed numerically by simulating a normalized standing wave with $\kappa=1, \omega=0.50$ and the centroid located at $x=0$ (see Columns 2-5 of Table I), where $\operatorname{err}_{2}$ and $\operatorname{err}_{\infty}$ are the $l^{2}$ and $l^{\infty}$ errors, respectively. The centroid position $q(t)$ does not change at all until $t=100$; see Column 6 of Table I. We have also shown there that $\mathcal{V}_{Q}, \mathcal{V}_{E}, \mathcal{V}_{P}$, measuring, respectively, the variation of charge, energy, and linear momentum at $t=100$ relative to the initial quantities, are all almost zero (see Columns 7-9), which demonstrates that the OS(4) method is able to keep the charge, energy, and linear momentum constant before the instability happens. (In fact, it will be 
shown later that this normalized standing wave is unstable and the instability appears at $t=11036$; see Fig. 6.) We can conclude that the OS(4) method is highly accurate and the numerical error is controlled only by the time step size $\tau$ for no approximation is used in space.

To perform the numerical study of the stability of NLD solitary waves, the employed numerical method is required

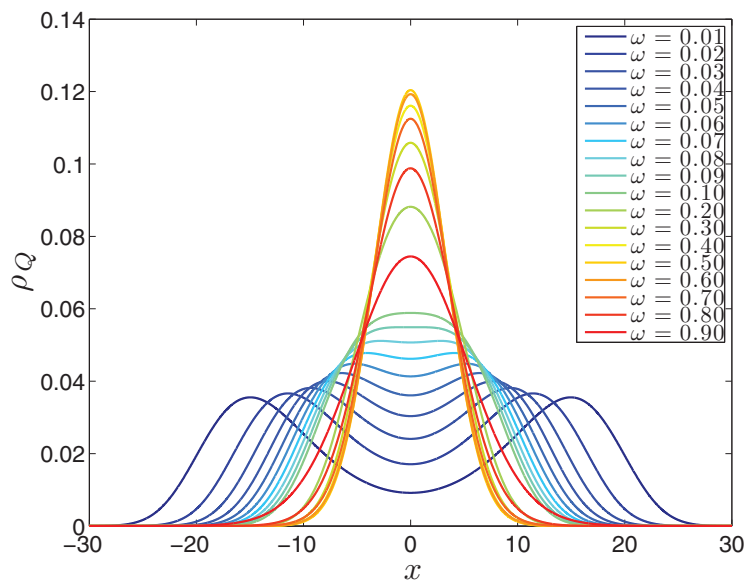

(a) $\kappa=0.1$

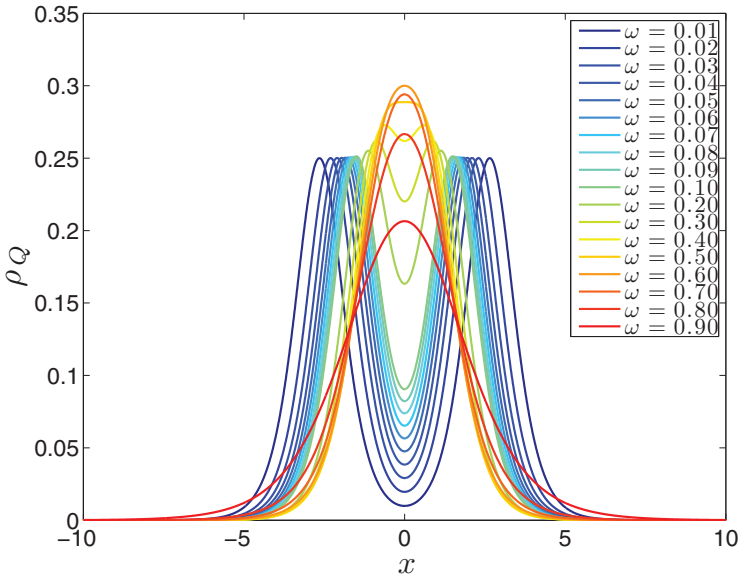

(c) $\kappa=1.0$

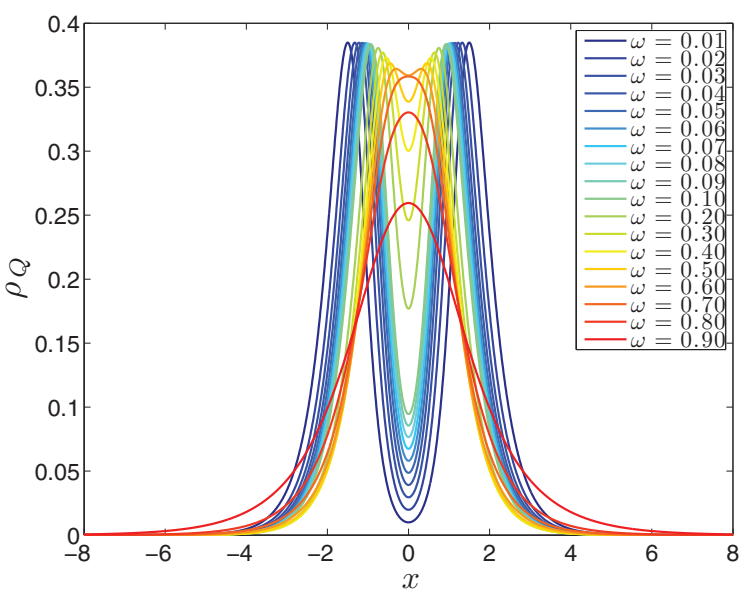

(e) $\kappa=2.0$ to be not only of high-order accuracy but also immune to the effect of artificial boundaries $X_{L, R}$. NRBC (75) used in the OS(4) method can avoid completely the numerical effect of $X_{L, R}$ on the stability of NLD solitary waves provided a relatively big domain $\left[X_{L}, X_{R}\right]$ is adopted, since it is transparent for outgoing waves and does not allow any waves to be pumped into the computational domain. In such situations,

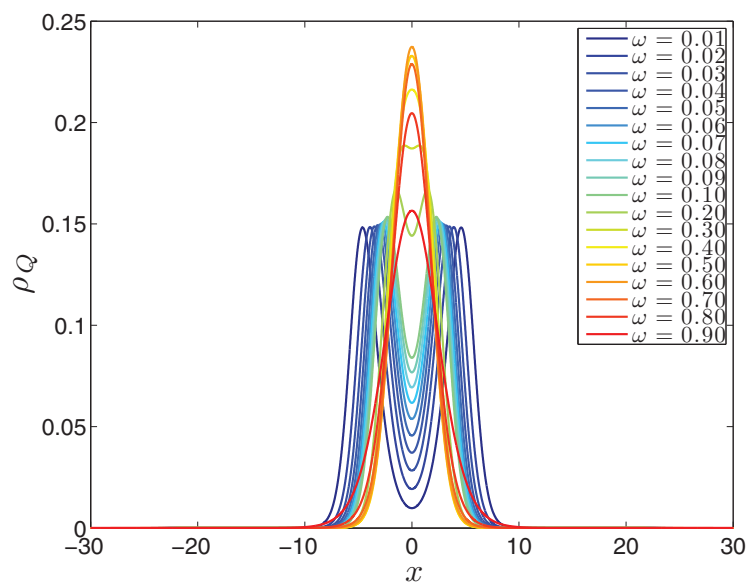

(b) $\kappa=0.5$

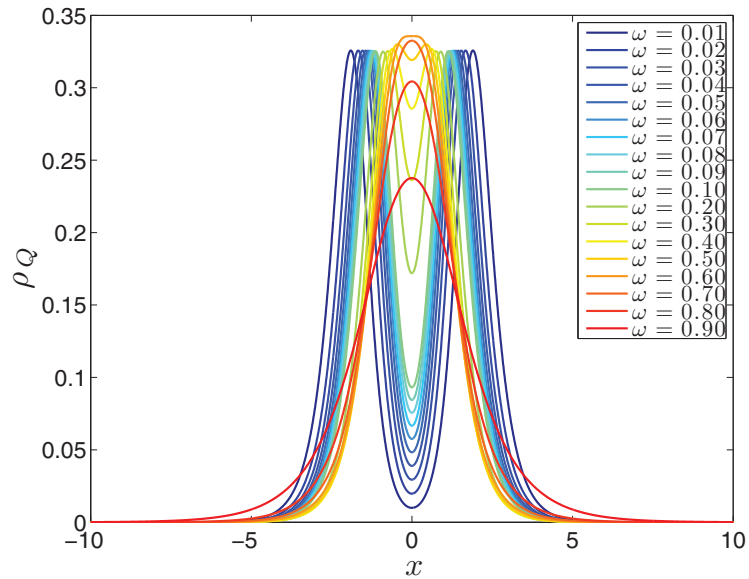

(d) $\kappa=1.5$

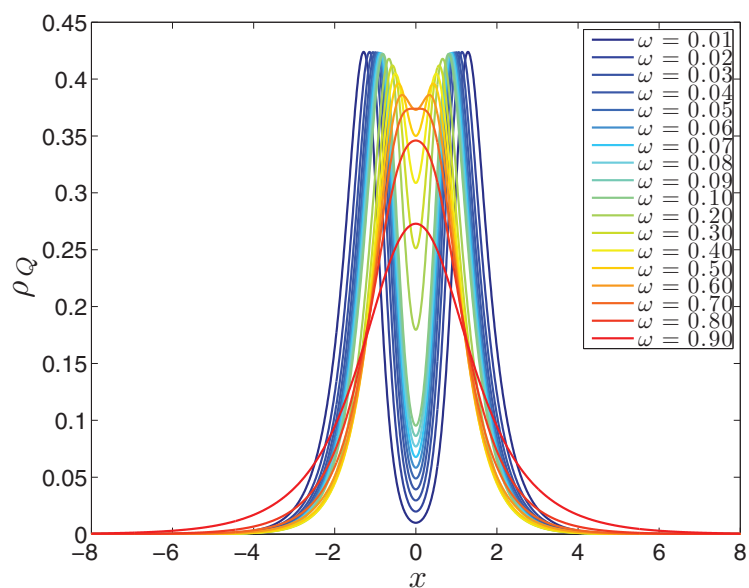

(f) $\kappa=2.4$

FIG. 4. (Color online) Typical profiles of the charge density $\rho_{Q}$ for various exponent powers (or the nonlinearity parameter) $\kappa$ and frequencies $\omega$. Smaller values of $\omega$ correspond to the outer double peaks and larger values to the inner single peak. 
TABLE II. The first column represents the computational domain lenghts $[-L, L]$. The other columns show the time at which the quantities $q, \operatorname{err}_{\infty}, \operatorname{err}_{2}, \mathcal{V}_{P}, \mathcal{V}_{E}$, and $\mathcal{V}_{Q}$ become larger than a given tolerance $\epsilon\left(=1.0 \times 10^{-3}\right)$. Here $q$ denotes the centroid position of the charge density and $\tau=0.025$.

\begin{tabular}{|c|c|c|c|c|c|c|}
\hline$L$ & $q$ & $\operatorname{err}_{\infty}$ & $\mathrm{err}_{2}$ & $\mathcal{V}_{P}$ & $\mathcal{V}_{E}$ & $\mathcal{V}_{Q}$ \\
\hline \multicolumn{7}{|c|}{ Two-hump wave with $\kappa=1$ and $\omega=0.1$} \\
\hline 50 & 147 & 121 & 121 & 135 & 131 & \\
\hline 75 & 146 & 122 & 122 & 135 & 132 & \\
\hline 100 & 146 & 122 & 122 & 134 & 132 & \\
\hline 125 & 146 & 122 & 120 & 135 & 139 & \\
\hline 150 & 145 & 122 & 122 & 133 & 132 & \\
\hline \multicolumn{7}{|c|}{ One-hump wave with $\kappa=1$ and $\omega=0.5$} \\
\hline$\overline{50}$ & 7373 & 6585 & 6614 & 6580 & 6601 & 6921 \\
\hline 75 & 9552 & 8728 & 8724 & 8720 & 8876 & 9177 \\
\hline 100 & 11036 & 9935 & 9937 & 9930 & 9930 & 10412 \\
\hline 125 & 12905 & 11673 & 11670 & 11672 & 11670 & 12183 \\
\hline 150 & 14641 & 13561 & 13560 & 13560 & 13560 & 14104 \\
\hline
\end{tabular}

we can also prove easily that the OS(4) method conserves the total charge. In summary, the proposed OS(4) method with $\mathrm{NRBC}$ is very appropriate and will be used for investigating the stability of NLD solitary waves.

\section{NUMERICAL RESULTS}

In accordance with the theoretical results, we consider merely the normalized NLD solitary waves; i.e., the charge is fixed to be $Q \equiv 1$. For such normalized NLD waves, only the frequency $\omega$ can be adjusted to get different profiles if fixing the mass $m=1$ and the exponent power (or the nonlinearity parameter) $\kappa$. For $\kappa=0.1,0.5,1.0,1.5,2.0,2.4$, Fig. 4 plots the profile transition of charge density $\rho_{Q}$ when $\omega$ increases from 0.01 to 0.9 . It is clearly observed there that, as the frequency increases, the charge density is transmitted from a two-hump profile to a one-hump profile during which the valley of the two-hump wave rises until the one-hump wave is

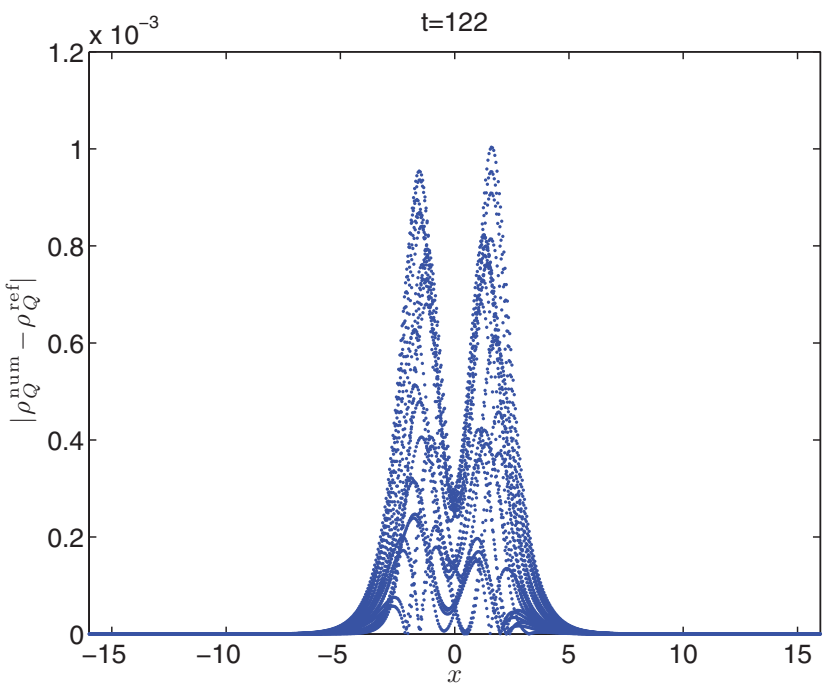

formed and then disappears; the maximum height of the peak of the one-hump wave is larger than that of the two-hump wave for $\kappa=0.1,0.5,1.0$, comparable for $\kappa=1.5$ and less than for $\kappa=2.0,2.5$. Actually it has been proved that the charge density has either one hump or two humps under the pure scalar self-interaction and also conjectured that there is a connection between the stability and the multihump structure [11,23]. In the following we will use the OS(4) method with NRBC to study such stability of normalized NLD waves and determine the range of $\omega$ in which the NLD solitary waves are stable or unstable for a given nonlinearity (or exponent power) $\kappa$. For simplicity, we only consider here the standing waves with the centroid located at $x=0$.

\section{A. $\kappa=1$}

In this section, we present the numerical results for the Soler model [16], i.e., $\kappa=1$. The first numerical simulation

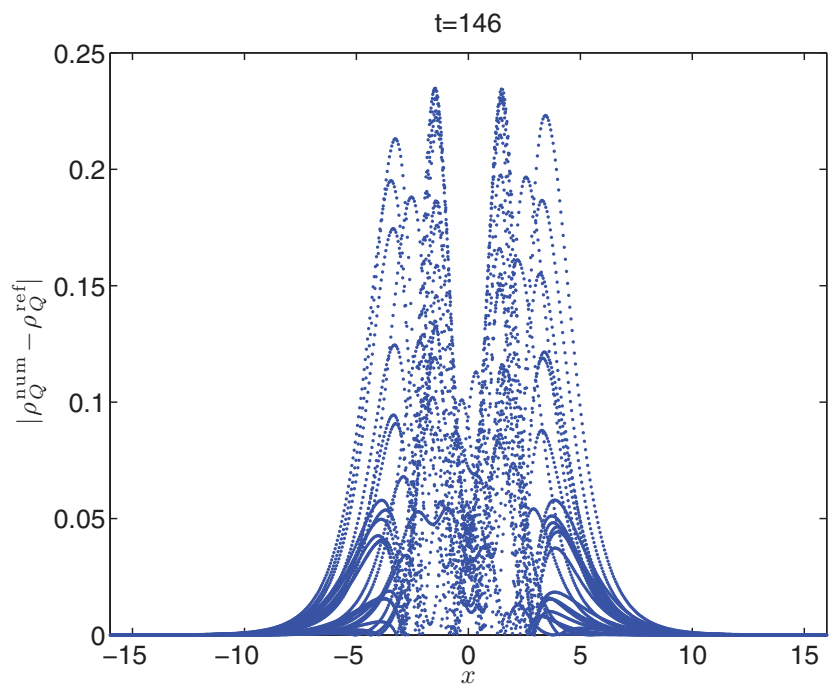

FIG. 5. (Color online) Unstable two-hump solitary wave with $\kappa=1$ and $\omega=0.1$ : Snapshot of the difference of the charge density between the numerical solution and the reference solution at $t=t_{e}=122$ and $t=t_{c}=146$ (left and right panels, respectively). Here $\tau=0.025$ and $L=100$. For each mesh point for $x$, there is only one value of the absolute difference. Due to the instability the wave breaks up into several waves, which seem to be represented by curves because of the very fine mesh. 

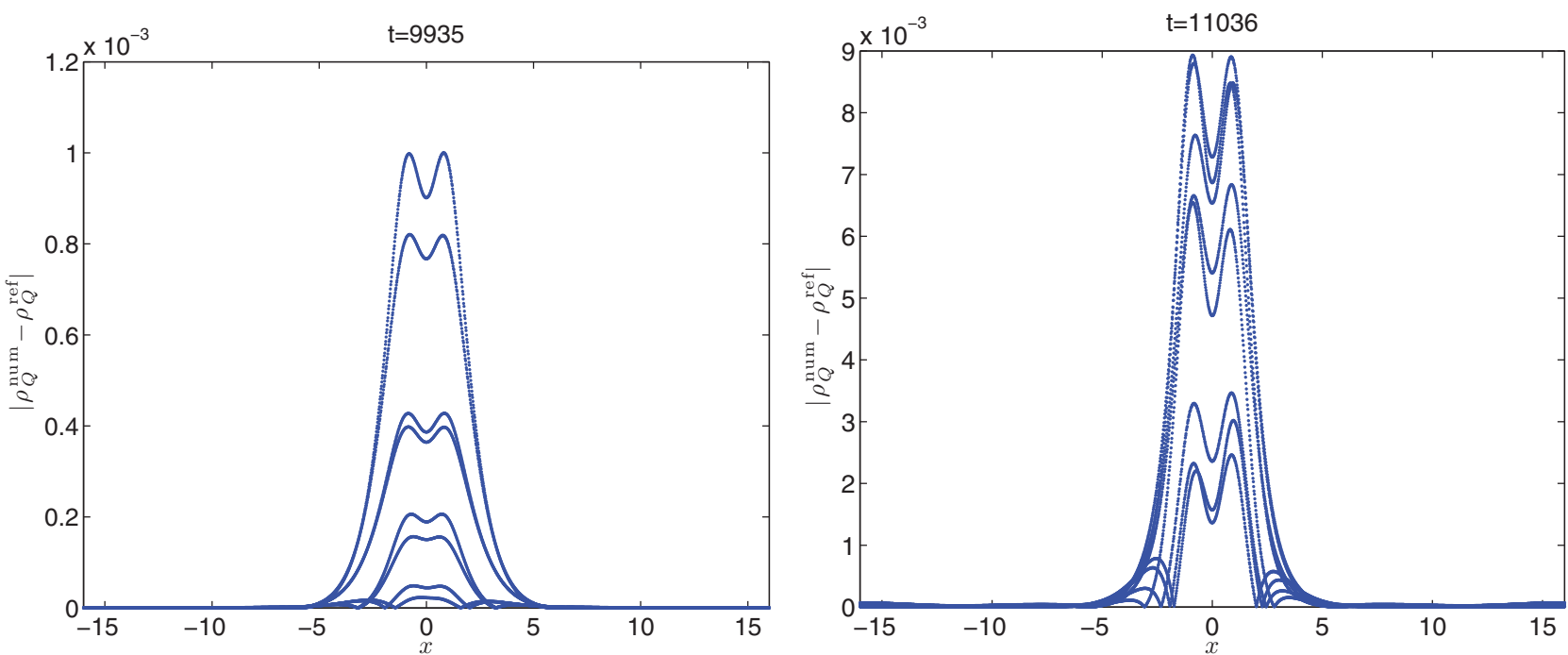

FIG. 6. (Color online) Unstable one-hump solitary wave with $\kappa=1$ and $\omega=0.5$ : Snapshot of the difference of the charge density between the numerical solution and the reference solution at $t=t_{e}=9935$ and $t=t_{c}=11036$, (left and right panels, respectively). Here $\tau=0.025$ and $L=100$. The comment about the very fine mesh in Fig. 5 holds here as well.

is performed using the OS(4) method for the two-hump wave with $\omega=0.1$; see Fig. 4(c). A large computational domain $[-L, L]$ (i.e., $X_{L}=-L, X_{R}=L$ ) is set with $L=100$. The time spacing, the parameter controlling the numerical error, is taken to be $\tau=0.025$. That is, the numerical error introduced by the OS(4) method at each time step is about $\tau^{4} \simeq$ 3.91E-07. However, the numerical error often accumulates slowly over time. If the solitary wave is unstable, such a slowly accumulated numerical error will be amplified in a relatively short period, after that the wave will change its position, which implies that the instability happens. This indeed occurs when $\omega=0.1$; see Row 5 of Table II. There we have shown the instants of time at which the monitored quantities, $q$, $\operatorname{err}_{\infty}, \operatorname{err}_{2}$, $\mathcal{V}_{P}, \mathcal{V}_{E}$, become larger than a given tolerance $\epsilon(=1.00 \mathrm{E}-03$ here). It can be seen that $\operatorname{err}_{\infty}$, $\operatorname{err}_{2}, \mathcal{V}_{E}, \mathcal{V}_{P}$, and $q$ increase over $\epsilon$ in sequence. We denote the instant at which the centroid position $q$ (resp. err ${ }_{\infty}$ ) becomes larger than $\epsilon$ by $t_{c}$ (resp. $t_{e}$ ). In Fig. 5 we plot the difference of the charge density between the numerical solution and the reference solution at $t_{e}=122$ and $t_{f}=146$, respectively. Meanwhile, the history of $q$ and err $_{\infty}$ is displayed in Fig. 7(a). It is observed there that, although the accumulated numerical error is larger than $\epsilon$ at $t_{e}=122$, the NLD wave still preserves its two-hump shape and its centroid hardly wavers from the initial position; after that, $\operatorname{err}_{\infty}$ increases quickly, soon the wave loses its shape, many waves are then generated, and the centroid moves from $x=0$ over $\epsilon$ at $t_{f}=146$. Hereafter, we define $t_{c}$ to be the moment at which the instability sets in. As shown clearly in Fig. 5, the entire process from $\operatorname{err}_{\infty}>\epsilon$ to $q>\epsilon$ develops very fast because it takes place only in the central area (around the initial centroid position $x=0$ ). This is also confirmed by numerical simulations within the domain $[-L, L]$ of different length, say,

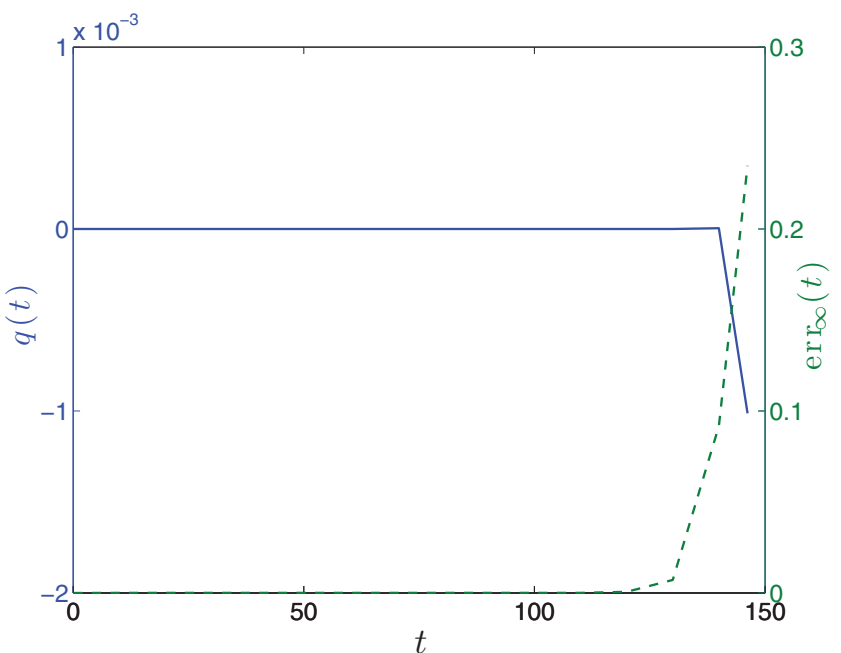

(a) $\omega=0.1$

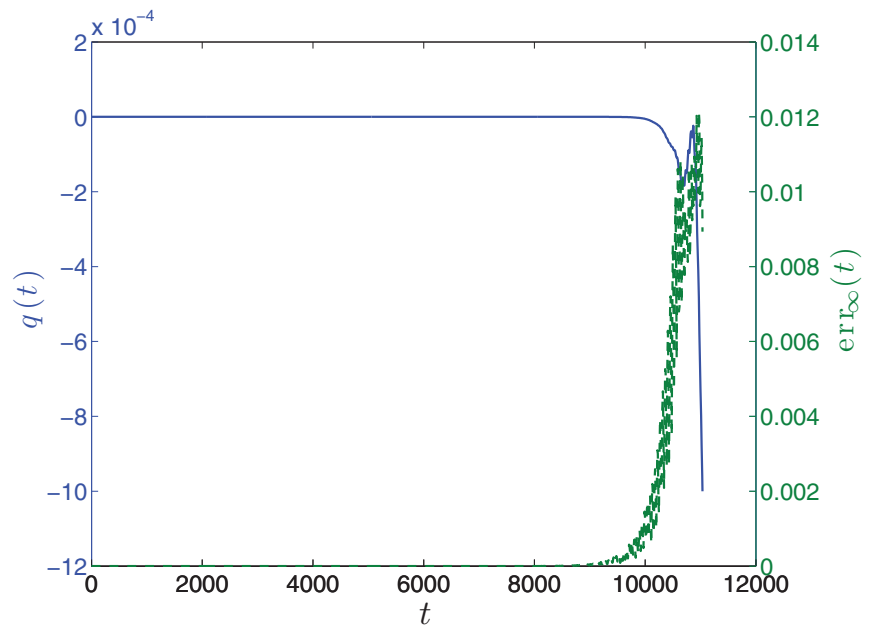

(b) $\omega=0.5$

FIG. 7. (Color online) Plots of the centroid position $q(t)$ (solid line) and the $l^{\infty}$ error err $\infty$ (dashed line) vs time for $\kappa=1$. Here $\tau=0.025$ and $L=100$. 
$L=50,75,100,125,150$, which reveal that instants of time at which the monitored quantities become larger than $\epsilon$ are nearly independent of the domain length; see Rows 2-7 of Table II. During the process, no charge is radiated out from the central area, and thus the total charge is conserved, e.g., at $t_{f}=146 \mathcal{V}_{Q} \simeq 2.23 \mathrm{E}-14$ for $L=75$, and $\mathcal{V}_{Q} \simeq 9.66 \mathrm{E}-15$ for $L=100$.

The second numerical simulation is performed for the one-hump wave with $\omega=0.5$; see Fig. 4(c). The setup of the OS(4) method for simulating the two-hump wave with $\omega=0.1$ is used. We plot the difference of the charge density between the numerical solution and the reference solution at $t_{e}=9935$ and $t_{f}=11036$ in Fig. 6 as well as the history of $q$ and $\operatorname{err}_{\infty}$ in Fig. 7(b). Therefore this one-hump wave is considered to be unstable. However, contrary to the fast process occurring only in the central area when $\omega=0.1$, the entire process from $\operatorname{err}_{\infty}>\epsilon$ to $q>\epsilon$ develops very slowly when $\omega=0.5$. As demonstrated by Figs. 6 and 7(b), the reason for such a slow process is the following: Although many waves of small amplitude are generated because of the instability, the wave is unstable only if enough generated waves move outside the computational domain. This is further confirmed by numerical simulations within domains of different lengths, the results of which can be found in Rows 8-13 of Table II. Those results show that the variation of charge $\mathcal{V}_{Q}$ decreases by $\epsilon$ before the instability occurs at $t_{c}$; and $t_{c}$ linearly depends on $L$ as plotted in Fig. 8 .

We have shown above that the OS(4) method with NRBC is capable of capturing the instability regardless of whether it occurs quickly or slowly. When the time step size $\tau$, the only parameter controlling the numerical error, decreases from 0.025 to 0.0125 , we have a very small change of $t_{c}$, e.g., $t_{c}=146$ (resp. $\left.t_{c}=11306\right)$ for $\tau=0.025$ and $t_{c}=148$ (resp. $\left.t_{c}=11278\right)$ for $\tau=0.0125$ when $\omega=0.10$ (resp. $\left.\omega=0.50\right)$. Consequently, the methodology to determine the stable range

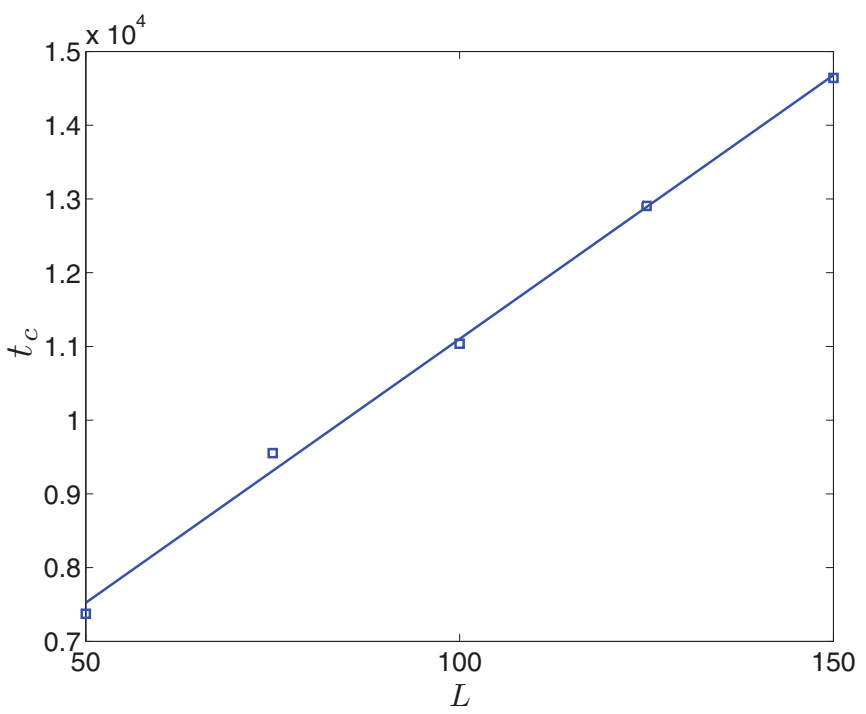

FIG. 8. (Color online) Plot of $t_{c}$ at which the centroid position $q(t)$ becomes larger than $\epsilon(=1.00 \mathrm{E}-03$ here $)$ with respect to $L$ for $\kappa=1$ and $\omega=0.5$. The computational domain is $[-L, L]$ and five different lengths are tested. The concrete data are given in Table II. Here $\tau=0.025$.

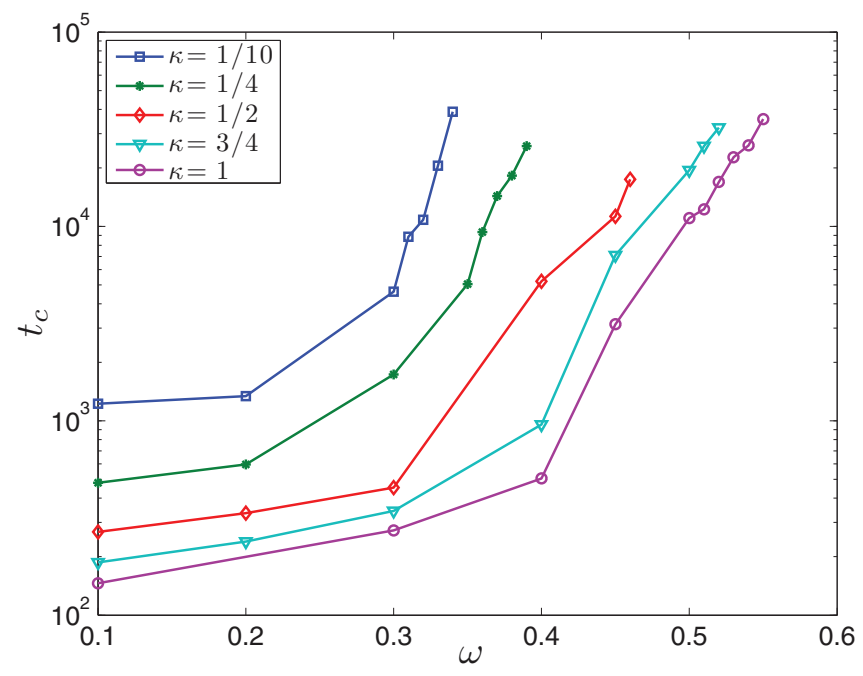

FIG. 9. (Color online) Plots of the instant $t_{c}$ against the frequency $\omega$ for $0<\kappa \leqslant 1$. The value of $\kappa$ increases from left to right.

for $\omega$ [denoted by $\Omega_{\kappa}$, being a subset of $(0,1)$ ] in which the NLD waves are stable, is to use the OS(4) method with $\tau=0.025$ and $L=100$ to simulate the wave with the frequency $\omega_{0}$. If the centroid position $q(t)$ is always less than the given tolerance $\epsilon$ before a prescribed final time $t_{\text {fin }}$, then $\omega_{0} \in \Omega_{\kappa}$, otherwise the NLD wave with $\omega_{0}$ is unstable, i.e., $\omega_{0} \in(0,1) \backslash \Omega_{\kappa}$. For the sake of confidence in our results, $t_{\text {fin }}$ should be long enough, and we choose $t_{\text {fin }}=40000$ in this work.

Our numerical simulations reveal that $\Omega_{1}=[0.56,1)$. When the frequency approaches 0.56 (the lower end of $\Omega_{1}$ ), the instant of instability $t_{c}$ increases exponentially; see Fig. 9.

\section{B. $0<\kappa<1$}

For $\kappa \in(0,1)$, we find the stable region $\Omega_{\kappa}$ for $\omega$ as follows: $\Omega_{1 / 10}=[0.35,1), \Omega_{1 / 4}=[0.40,1), \Omega_{1 / 2}=[0.47,1)$, and $\Omega_{3 / 4}=[0.53,1)$, all of which are left-closed and rightopen intervals with the same right end of 1 . Moreover, it is observed that the left end of $\Omega_{\kappa}$ increases monotonically as $\kappa$ increases from 0 to 1 , and the limit is about 0.60 for larger values of $\kappa$; see Fig. 10 .

\section{C. $1<\kappa<2$}

For $\kappa \in(1,2)$, we find two types of stable region $\Omega_{\kappa}$ : the first type is a left-closed and right-open interval with the left end around 0.60 and the right end at 1 , e.g., $\Omega_{5 / 4}=$ $[0.58,1)$ and $\Omega_{7 / 4}=[0.89,1)$; the second type consists of two disjoint intervals, e.g., $\Omega_{11 / 8}=[0.58,0.67] \cup[0.77,1)$ and $\Omega_{3 / 2}=[0.59,0.64] \cup[0.85,1)$. In Fig. 11 we plot $t_{c}$ against $\omega$ for $1<\kappa<2$, where $t_{c}$ is not available for the stable NLD waves and we use $t_{\text {fin }}=40000$ instead. That is, the flat part of the curve with a value of 40000 corresponds to the waves in the stable region. It can be easily observed there that: When the exponent power $\kappa$ is slightly larger than 1, we have a large stable region of the first type; when we keep increasing $\kappa$, this large stable region is divided into two small intervals located around the left end and the right end, respectively, which form together the stable region of the second type, 


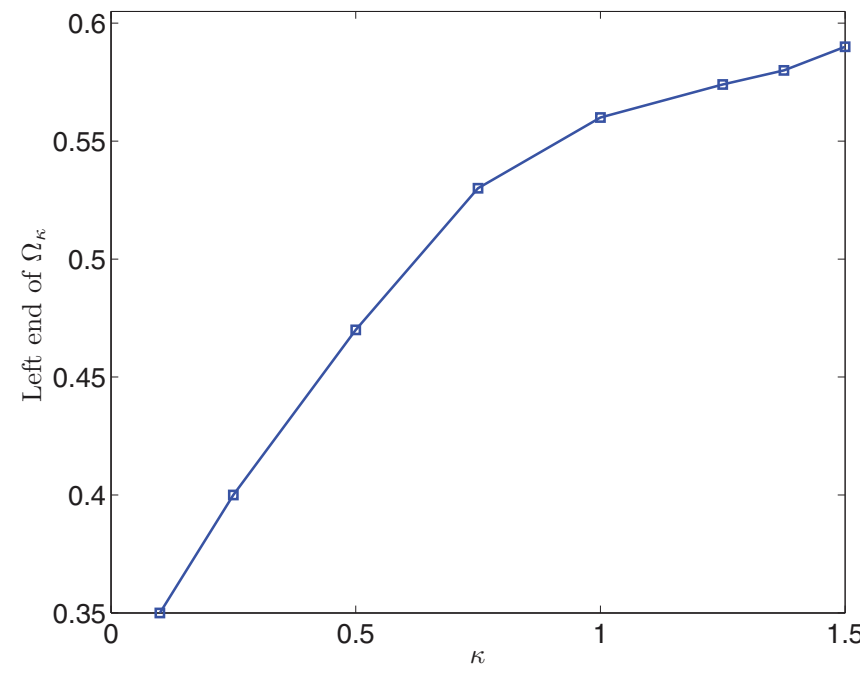

FIG. 10. (Color online) Plot of the left end of the stable region $\Omega_{\kappa}$ for $0<\kappa \leqslant 3 / 2$.

one closed interval with the left end around 0.60 and the other left-closed and right-open interval with the right end of 1 ; when $\kappa$ approaches 2 , the small interval around 0.60 disappears, and then we have again the stable region of the first type but of much shorter length. As the frequency approaches the left end of $\Omega_{\kappa}$, the instant of the instability $t_{c}$ increases exponentially. In the case of a stable region of the second type, $t_{c}$ for the unstable NLD waves with the frequency $\omega$ between the two disjoint intervals oscillates in $\omega$ and decreases monotonically in $\kappa$ for a given frequency.

\section{D. $\kappa \geqslant 2$}

For $\kappa \geqslant 2$, the stable region exists only for $\kappa$ slightly larger than as well as equal to 2 , e.g., $\Omega_{2}=[0.92,1), \Omega_{2.1}=$ $[0.93,0.97]$, and $\Omega_{2.2}=[0.93,0.94]$. For larger $\kappa$, the NLD

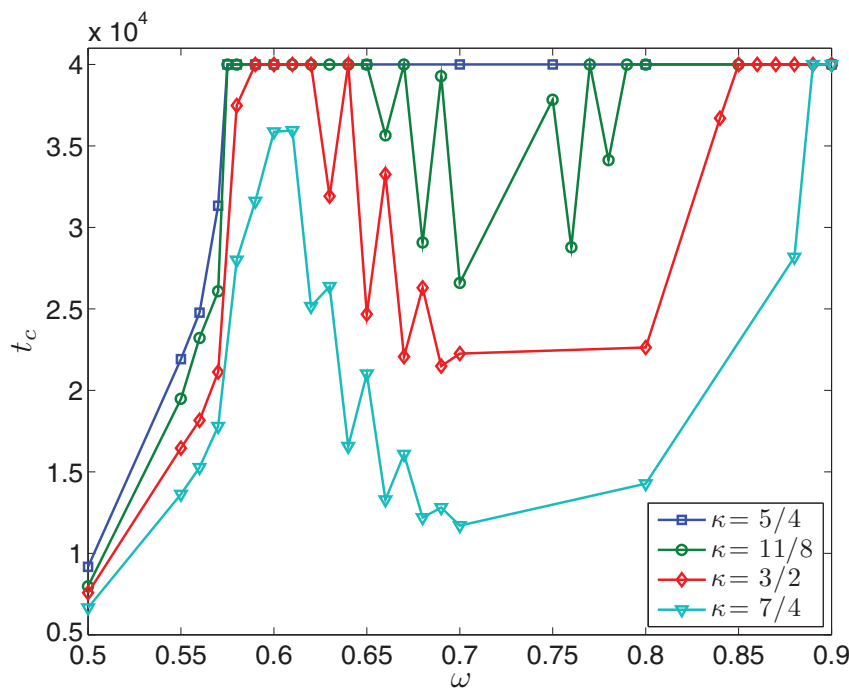

FIG. 11. (Color online) Plot of $t_{c}$ vs $\omega$ for $1<\kappa<2$. For the NLD waves in the stable region, $t_{c}$ is not available, and we use $t_{\text {fin }}=40000$ instead. That is, the flat parts of the curve with a value of 40000 correspond to the waves in the stable region. The value of $\kappa$ increases from top to bottom.

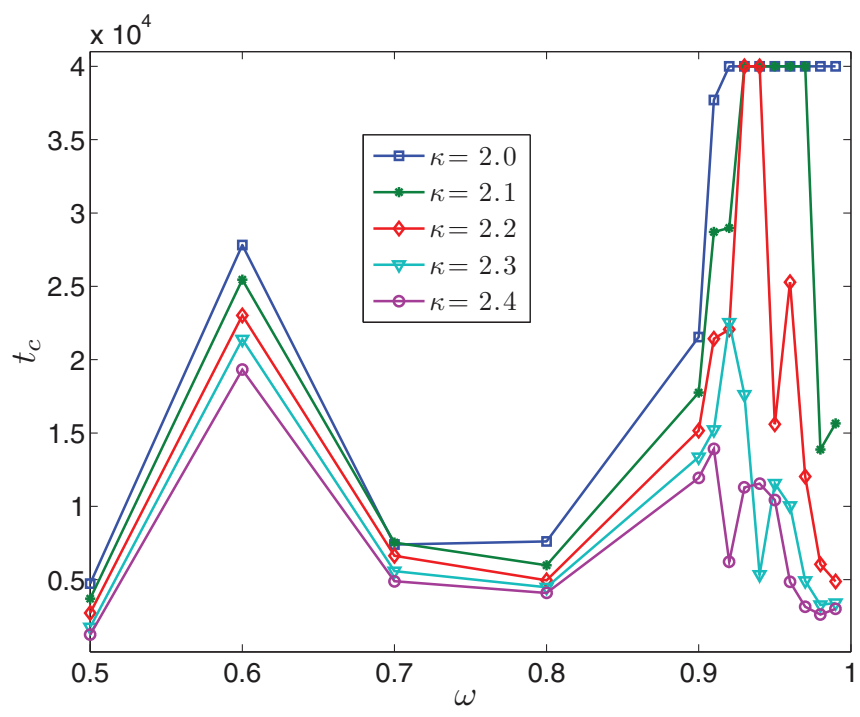

FIG. 12. (Color online) Plot of $t_{c}$ vs $\omega$ for $\kappa \geqslant 2$. For the NLD waves in the stable region, $t_{c}$ is not available, and we use $t_{\text {fin }}=40000$ instead. That is, the flat parts of the curves with a value of 40000 correspond to the waves in the stable region. The value of $\kappa$ increases from top to bottom.

waves are unstable for all $\omega \in(0,1)$, e.g., $\Omega_{2.3}=\Omega_{2.4}=\varnothing$. In Fig. 12 we plot $t_{c}$ against $\kappa$ and see that the instant of instability $t_{c}$ increases exponentially as $\omega$ approaches the left end of $\Omega_{\kappa}$, and decreases monotonically in $\kappa$ for a given frequency.

\section{E. Discussion}

According to the discovered stable region $\Omega_{\kappa}$ for $\kappa>0$ and Fig. 4, we can conclude that all stable NLD waves are of one-hump profile, which gives a positive answer to the conjecture put forth in $[11,23]$, i.e., the NLD waves of twohump structure are unstable. This is also in accordance with numerical observations in Ref. [24], which imply that the twohump NLD solitary waves may collapse during scattering (i.e., after collision they stop being solitary waves), whereas the collapse phenomena cannot be generally observed in collisions of the one-hump NLD solitary waves.

When the exponent power $\kappa$ (denoting the strength of nonlinearity) increases, the stable region $\Omega_{\kappa}$ narrows. For a given $\omega$ in the unstable region, the moment of instability $t_{c}$ decreases monotonically with increasing $\kappa$; see, e.g., Figs. 11 and 12. Particularly, for $\omega=0.1$, we find that $t_{c}$ is inversely proportional to $\kappa$; see Fig. 13.

\section{SUMMARY}

In this paper we reviewed various variational methods that had been put forward to determine possible criteria for the exact solitary wave solutions to the NLD equation to be unstable. We showed that these methods yield inconsistent results (in contrast to the NLS equation for which the results of all these methods agree): The arguments of Bogolubsky suggested that for $\omega$ less than a critical value $\omega_{B} \approx 0.7$, which is practically independent of $\kappa$, the solitary waves should be unstable to slight changes in $\omega$ for fixed charge $Q$. An argument 


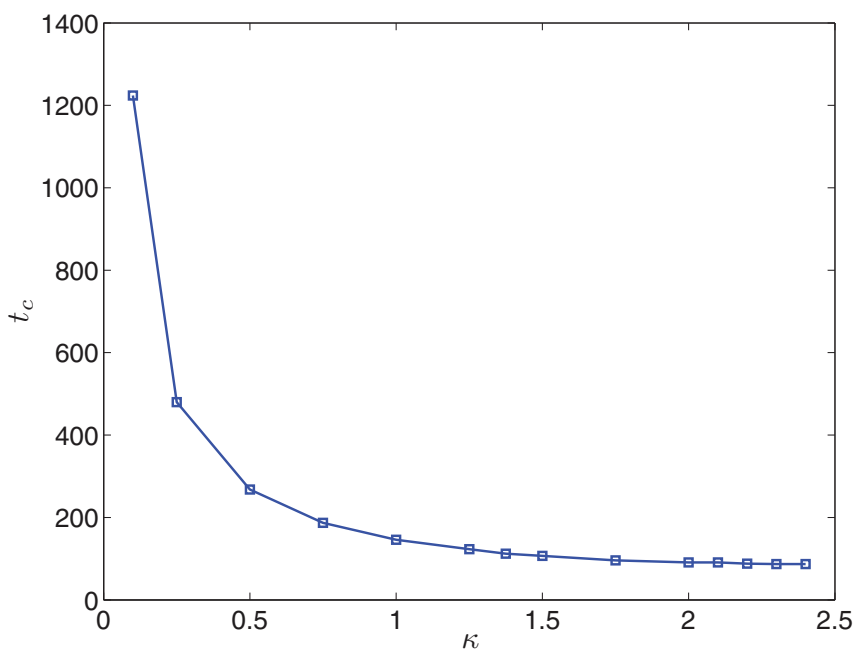

FIG. 13. (Color online) Plot of $t_{c}$ against $\kappa$ for unstable NLD waves with $\omega=0.1$.

based on scale transformations suggested that the solitary wave solutions are unstable for all $\kappa>1$. The Vakhitov-Kolokolov criterion suggested that for $\kappa<2$ all solitary waves are stable, and for $\kappa>2$ there is a region of $\omega$ below a curve $\omega(\kappa)$ where the solitary waves are suggested to be stable. As the above suggestions yielded inconsistent results, we performed extensive numerical simulations in order to determine the stability regions $\Omega_{\kappa}$ for $\omega$. For $0<\kappa<1$ the stability regions are left-closed and right-open intervals with the same right end of 1 , while the left end increases with $\kappa$. For $\kappa=1$ the stability interval is $[0.56,1)$. For $1<\kappa<2$ we find two types of $\Omega_{\kappa}$ : The first one is a left-closed and right-open interval with the left end around 0.60 and the right end at 1 . The second type consists of two disjoint intervals. For $\kappa=2$ there is a stable region just below 1 . For $\kappa>2$ a very narrow stable region exists only for $\kappa$ slightly larger than 2 . For $0<\kappa<1$ the time $t_{c}$ when an instability sets in, increases exponentially with $\omega$ while the stable region is approached. For $1<\kappa<2$, $t_{c}$ is a very complicated function of $\omega$ in the instability regions and $t_{c}$ decreases monotonically with increasing $\kappa$. The stability of the solitary waves depends on their profile, i.e., on the shape of the charge density as a function of $x$. All stable waves have a one-hump profile, but not all one-hump waves are stable. All waves with two humps are unstable. An open issue is the study of collisions of NLD solitary waves with different $\kappa$ values. Our results are relevant to understanding nonlinear phenomena in Bose-Einstein condensates in honeycomb lattices [1,2] and optical binary waveguides $[3,4]$ as well as nonlinear dynamics [5] and diffraction in photonic graphene [6].

\section{ACKNOWLEDGMENTS}

This work was performed in part under the auspices of the United States Department of Energy. F.G.M., F.C., and A.S. would like to thank the Santa Fe Institute for its hospitality during the completion of this work. We also thank Prof. A. Comech for his useful comments on a draft of this paper. S.S. acknowledges financial support from the National Natural Science Foundation of China (Nos. 11101011, 11471025, 11421101) and the Specialized Research Fund for the Doctoral Program of Higher Education (No. 20110001120112). N.R.Q. acknowledges financial support from the Humboldt Foundation through Research Fellowship for Experienced Researchers SPA 1146358 STP and by the MICINN through FIS2011-24540, and by Junta de Andalucia under Projects No. FQM207, No. P06-FQM-01735, and No. P09-FQM-4643. F.G.M. acknowledges the hospitality of the Mathematical Institute of the University of Seville (IMUS) and of the Theoretical Division and Center for Nonlinear Studies at Los Alamos National Laboratory, financial support by the Plan Propio of the University of Seville, and by the MICINN through FIS2011-24540. A.K. acknowledges financial support from Department of Atomic Energy, Government of India through a Raja Ramanna Fellowship.
[1] L. H. Haddad and L. D. Carr, Physica D 238, 1413 (2009).

[2] L. H. Haddad and L. D. Carr, Europhys. Lett. 94, 56002 (2011).

[3] T. X. Tran, S. Longhi, and F. Biancalana, Ann. Phys. 340, 179 (2014).

[4] T. X. Tran, X. N. Nguyen, and D. C. Dung, J. Opt. Soc. Am. B 31, 1132 (2014).

[5] O. Bahat-Treidel and M. Segev, Phys. Rev. A 84, 021802(R) (2011).

[6] M. J. Ablowitz and Y. Zhu, Opt. Lett. 36, 3762 (2011).

[7] S. Y. Lee, T. K. Kuo, and A. Gavrielides, Phys. Rev. D 12, 2249 (1975).

[8] Y. Nogami and F. M. Toyama, Phys. Rev. A 45, 5258 (1992).

[9] D. J. Gross and A. Neveu, Phys. Rev. D 10, 3235 (1974).

[10] W. Thirring, Ann. Phys. 3, 91 (1958).

[11] F. Cooper, A. Khare, B. Mihaila, and A. Saxena, Phys. Rev. E 82, 036604 (2010).

[12] I. L. Bogolubsky, Phys. Lett. A 73, 87 (1979).

[13] G. H. Derrick, J. Math. Phys. 5, 1252 (1964).
[14] A. Comech, M. Guan, and S. Gustafson, Ann. Inst. Henri Poincaré 31, 639 (2014); arXiv:1203.3859, and references therein.

[15] N. G. Vakhitov and A. A. Kolokolov, Radiophys. Quantum Electron. 16, 783 (1973).

[16] A. Alvarez and M. Soler, Phys. Rev. Lett. 50, 1230 (1983).

[17] A. Alvarez and B. Carreras, Phys. Lett. A 86, 327 (1981).

[18] F. G. Mertens, N. R. Quintero, F. Cooper, A. Khare, and A. Saxena, Phys. Rev. E 86, 046602 (2012).

[19] F. Cooper, A. Khare, N. R. Quintero, F. G. Mertens, and A. Saxena, Phys. Rev. E 85, 046607 (2012).

[20] J. Xu, S. H. Shao, and H. Z. Tang, J. Comput. Phys. 245, 131 (2013).

[21] A. T. Sornborger and E. D. Stewart, Phys. Rev. A 60, 1956 (1999).

[22] G. Strang, SIAM J. Numer. Anal. 5, 506 (1968).

[23] J. Xu, S. H. Shao, H. Z. Tang, and D. Y. Wei, arXiv:1311.7453 [nlin.SI] [Commun. Math. Sci. (to be published)].

[24] S. H. Shao and H. Z. Tang, Phys. Lett. A 345, 119 (2005). 